\title{
The ISO Long Wavelength Spectrometer line spectrum of VY Canis Majoris and other oxygen-rich evolved stars ${ }^{\star}$
}

\author{
E. T. Polehampton ${ }^{1,2,3, \star \star}$, K. M. Menten ${ }^{1}$, F. F. S. van der Tak ${ }^{4,1}$, and G. J. White 2,5
}

\author{
1 Max-Planck-Institut für Radioastronomie, Auf dem Hügel 69, 53121 Bonn, Germany \\ 2 Space Science \& Technology Department, Rutherford Appleton Laboratory, Chilton, Didcot, Oxfordshire, OX11 0QX, UK \\ e-mail: edward.polehampton@stfc.ac.uk \\ 3 Department of Physics, University of Lethbridge, 4401 University Drive, Lethbridge, Alberta, T1J 1B1, Canada \\ 4 Netherlands Institute for Space Research (SRON), Landleven 12, 9747 AD Groningen, The Netherlands \\ 5 Department of Physics and Astronomy, The Open University, Milton Keynes, MK6 7AA, UK
}

Received 16 September 2009 / Accepted 10 November 2009

\section{ABSTRACT}

\begin{abstract}
Context. The far-infrared spectra of circumstellar envelopes around various oxygen-rich stars were observed using the ISO Long Wavelength Spectrometer (LWS). These have been shown to be spectrally rich, particularly in water lines, indicating a high $\mathrm{H}_{2} \mathrm{O}$ abundance.

Aims. We have examined high signal-to-noise ISO LWS observations of the luminous supergiant star, VY CMa, with the aim of identifying all of the spectral lines. By paying particular attention to water lines, we aim to separate the lines due to other species, in particular, to prepare for forthcoming observations that will cover the same spectral range using Herschel PACS and at higher spectral resolution using Herschel HIFI and SOFIA.

Methods. We have developed a fitting method to account for blended water lines using a simple weighting scheme to distribute the flux. We have used this fit to separate lines due to other species which cannot be assigned to water. We have applied this approach to several other stars which we compare with VY CMa.

Results. We present line fluxes for the unblended $\mathrm{H}_{2} \mathrm{O}$ and $\mathrm{CO}$ lines, and present detections of several possible $v_{2}=1$ vibrationally excited water lines. We also identify blended lines of $\mathrm{OH}$, one unblended and several blended lines of $\mathrm{NH}_{3}$, and one possible detection of $\mathrm{H}_{3} \mathrm{O}^{+}$.

Conclusions. The spectrum of VY CMa shows a detection of emission from virtually every water line up to $2000 \mathrm{~K}$ above the ground state, as well as many additional higher energy and some vibrationally excited lines. A simple rotation diagram analysis shows large scatter (probably due to some optically thick lines). The fit gives a rotational temperature of $670_{-130}^{+210} \mathrm{~K}$, and lower limit on the water column density of $(7.0 \pm 1.2) \times 10^{19} \mathrm{~cm}^{-2}$. We estimate a CO column density $\sim 100$ times lower, showing that water is the dominant oxygen carrier. The other stars that we examined have similar rotation temperatures, but their $\mathrm{H}_{2} \mathrm{O}$ column densities are an order of magnitude lower (as are the mass loss rates).
\end{abstract}

Key words. stars: AGB and post-AGB - stars: individual: VY Canis Majoris - supergiants - circumstellar matter

\section{Introduction}

Circumstellar envelopes form around red giant and supergiant stars during the later stages of their evolution. The mass loss from the stars results in an expanding envelope of material consisting of dust and molecules whose absorption and/or emission can be observed from infrared through radio wavelengths. In oxygen-rich stars with high mass loss rates, the cooling of the envelope is believed to be dominated by rotational lines from water $\left(\mathrm{H}_{2} \mathrm{O}\right)$ vapour (e.g. Justtanont et al. 1994), which has a rich far infrared (FIR) spectrum. The FIR range also contains the rotational transitions of other simple molecules and molecular ions.

The Infrared Space Observatory (ISO; Kessler et al. 1996) satellite has revolutionised the study of circumstellar envelopes

* Based on observations with ISO, an ESA project with instruments funded by ESA Member States (especially the PI countries: France, Germany, the Netherlands and the United Kingdom) with the participation of ISAS and NASA.

$\star \star$ Current address: Space Science Department, Rutherford Appleton Laboratory, UK by allowing access to all the spectral features in the midand far-infrared range. ISO spectra observed using the Short Wavelength Spectrometer (SWS; de Graaw et al. 1996) and Long Wavelength Spectrometer (LWS; Clegg et al. 1996) have previously been analysed, showing that the majority of the detected features in the FIR range are due to the rotational transitions of water (W Hya SWS: Neufeld et al. 1996; W Hya LWS: Barlow et al. 1996; R Cas LWS: Truong-Bach et al. 1999; VY CMa SWS: Neufeld et al. 1999).

In this paper, we present a dataset containing the ISO LWS spectra of the red supergiant star VY CMa, and several other evolved stars. The basic parameters of these spectra, such as identifications of lines over the wavelength range 45-196 $\mu \mathrm{m}$ and their fluxes are discussed here. We concentrate on the results from VY CMa, as it shows the spectrum with the highest signal to noise ratio and the line identifications are typical of several other stars in the sample. The SWS line spectrum for this star has previously been presented by Neufeld et al. (1999). The continuum emission of the SWS and LWS spectra has been modeled by Harwit et al. (2001) and the LWS line emission has been briefly described by Barlow (1999). VY CMa is thought to be in 
Table 1. Characteristics of the stars used in this paper.

\begin{tabular}{lccc}
\hline \hline Star name & $\begin{array}{c}\text { Distance } \\
(\mathrm{pc})\end{array}$ & $\begin{array}{c}\text { Mass loss rate } \\
\left(M_{\odot} \mathrm{yr}^{-1}\right)\end{array}$ & Ref. \\
\hline VY CMa & 1100 & $0.5-1 \times 10^{-4}$ & $(1),(2)$ \\
IK Tau & 300 & $4.9 \times 10^{-6}$ & $(3)$ \\
TX Cam & 390 & $2.6 \times 10^{-6}$ & $(3)$ \\
RX Boo & 160 & $2.2 \times 10^{-7}$ & $(4)$ \\
IRC+10011 (WX Psc) & 830 & $6 \times 10^{-6}$ & $(5)$ \\
R Cas & 107 & $3.4 \times 10^{-7}$ & $(6)$ \\
\hline
\end{tabular}

Notes. (1) Reid \& Menten (in preparation); Choi et al. (2008); (2) Decin et al. (2006); (3) Olivier et al. (2001); (4) Teyssier et al. (2006); (5) Decin et al. (2007); (6) Truong-Bach et al. (1999).

a late stage of stellar evolution, and appears extremely bright at infrared wavelengths. It has a mass loss rate estimated to be of the order of $5 \times 10^{-5}$ to $1 \times 10^{-4} M_{\odot} \mathrm{yr}^{-1}$ with a period of higher mass loss in the past (Decin et al. 2006). It is located at the edge of a molecular cloud (Lada \& Reid 1978), and has a distance derived from $\mathrm{H}_{2} \mathrm{O}$ and $\mathrm{SiO}$ masers of $\sim 1.1 \mathrm{kpc}$ from the Sun (Choi et al. 2008; Reid \& Menten, in preparation). In Sect. 3, we estimate the column densities of water and $\mathrm{CO}$ in the envelope of VY CMa to determine which is the dominant oxygen carrier. In Sect. 4, we compare the results from VY CMa with several other stars that were also observed with the ISO LWS. The characteristics of these stars are shown in Table 1.

\section{Observations and data reduction}

A complete set of uniformly processed LWS grating spectra (using ISO observing mode L01) have been uploaded to the ISO Data Archive ${ }^{1}$ as highly processed data products (HPDP; Lloyd et al. 2003). This allows us to make a detailed comparison of consistently and accurately calibrated spectral lines observed toward a sample of different stars. The objects, positions and ISO target dedicated time (TDT) numbers of the data we have used are shown in Table 2.

To check the accuracy of the standard HPDP pipeline processing for our individual objects, we downloaded and manually reduced the LWS L01 spectrum of VY CMa, comparing it with the HPDP data (to examine the effects of automatically removing glitches and dark current subtraction). Both the test observations and the HPDP were initially processed with the LWS off line pipeline (OLP) version 10.

In order to reduce the data by hand, we used the LWS Interactive Analysis (LIA; Lim et al. 2002) software to optimise the dark current subtraction for each LWS detector. The absolute responsivity correction was not adjusted interactively because the OLP data already contains a better method of calculating the corrections to that available in the LIA software. Glitches were carefully removed from each scan by hand using the ISO Spectral Analysis Package (ISAP; Sturm et al. 1998). In addition, forward and backward scans were compared and the regions where one direction was affected by the response time of the detectors were removed (this can occur when the grating was scanned in the direction of increasing detector response from the edge of each band). A correction for fringing was applied using the LWS defringing algorithm within ISAP. The forward and reverse scans for each detector were then averaged.

A careful comparison of the interactively reduced and HPDP spectra showed that there were only minor differences in the

${ }^{1}$ See www.iso.esac.esa.int/ida/ final strength of the lines. This is mainly due to the defringing step which was only performed in the HPDP processing for detectors that the pipeline judged significantly affected (in the case of VY CMa, only for detector SW5, whereas interactively we judged defringing to make most difference to detector LW4). Further differences that may cause problems are that in the HPDP data reduction, both scan directions were simply averaged together (without accounting for the transient response of the detectors) and deglitching was carried out automatically. However, neither of these appear to have led to the introduction of spurious features in the HPDP spectrum.

The largest difference between the two data reduction techniques is an improvement in the spectral shape of data from detector SW1 in the HPDP data. This is due to an additional correction in the HPDP pipeline that removes a double-peaked structure (with peaks at $\sim 45 \mu \mathrm{m}$ and $\sim 48 \mu \mathrm{m}$ ) that is believed to be due to spurious features in the SW1 relative spectral response function (Lloyd et al. 2003). Using this correction allows us to have much greater confidence in the spectral shape observed using SW1 between 43 and $50 \mu \mathrm{m}$.

In conclusion, we determined that the HPDP pipeline did not introduce spurious artifacts that could be mistaken for lines, and that it had superior calibration for detector SW1. Therefore, we have used only the HPDP data for all of the stars in our sample. We applied multiplicative shifts to individual detector data to align their continua by examining the overlap region between pairs of detectors. However, this was not important for the spectrum of VY CMa because the absolute flux calibration applied by the HPDP already gave very good agreement between adjacent detectors, with the only exception being SW1, which had an absolute flux $\sim 20 \%$ higher than SW2. For the other stars, the shifts were more often required for the long wavelength detectors (up to $\sim 30 \%$ ). In order to examine the line fluxes, we subtracted the continuum level by fitting a 3rd order polynomial baseline to the data from each detector independently.

Several well known spurious features that resemble lines remain in the spectra, and these are labeled in the plots presented here (e.g. features in absorption at $77 \mu \mathrm{m}$ using SW5 and $191 \mu \mathrm{m}$ using LW5, and features in emission at $107 \mu \mathrm{m}$ and $109 \mu \mathrm{m}$ using LW2; Grundy, private communication). Some of these lines occur in the overlap region between two detectors and so can be ignored by selecting the optimum wavelength ranges to use. To provide a further check that the line features we have identified are real, we compared the spectra to observations of the asteroid Ceres, which should not contain any spectral line features (but does contain the spurious features).

Where there were multiple LWS observations of the sample stars that showed good agreement, these were co-added to increase the signal to noise ratio. This applied to IRC+10011 and RX Boo (see Table 2). Although several other observations were also available for R Cas (TDTs 26300712, 56801715 and 56801552), we have used only the one with longest integration time (TDT 56801440).

The LWS beam size was $\sim 80^{\prime \prime}$, and the spectral resolution was $0.29 \mu \mathrm{m}$ for detectors SW1-SW5 and $0.6 \mu \mathrm{m}$ for detectors LW1-LW5 (Gry et al. 2003). The RMS noise in the spectra varies for the different stars, but is roughly $(10-20) \times 10^{-20} \mathrm{~W} \mathrm{~cm}^{-2} \mu \mathrm{m}^{-1}$ in the short wavelength detectors (measured between 87 and $89.5 \mu \mathrm{m}$ ), and $(0.5-2.2) \times 10^{-20} \mathrm{~W} \mathrm{~cm}^{-2} \mu \mathrm{m}^{-1}$ in the long wavelength detectors (measured between 140 and $142 \mu \mathrm{m}$ ). The wavelength bin width is $0.04 \mu \mathrm{m}$ for the short wavelength detectors and $0.13 \mu \mathrm{m}$ for the long wavelength detectors. The final spectra before continuum subtraction are shown in Fig. 1, plotted as $\lambda^{4} f_{\lambda}$. In this 
Table 2. Log of the L01 observations used in this paper.

\begin{tabular}{lcccc}
\hline \hline Star & ISO TDT & Integration time (s) & Date & Coordinates (J2000) \\
\hline VY CMa & 73502338 & 1930 & $20-11-97$ & $07^{\mathrm{h}} 22^{\mathrm{m}} 58.21^{\mathrm{s}},-25^{\circ} 46^{\prime} 02.6^{\prime \prime}$ \\
IK Tau & 65601707 & 3430 & $02-09-97$ & $03^{\mathrm{h}} 53^{\mathrm{m}} 28.86^{\mathrm{s}}, 11^{\circ} 24^{\prime} 22.3^{\prime \prime}$ \\
TX Cam & 69501069 & 3428 & $10-10-97$ & $05^{\mathrm{h}} 00^{\mathrm{m}} 50.37^{\mathrm{s}}, 56^{\circ} 10^{\prime} 52.5^{\prime \prime}$ \\
RX Boo & 62300514 & 1328 & $31-07-97$ & $14^{\mathrm{h}} 24^{\mathrm{m}} 11.62^{\mathrm{s}}, 25^{\circ} 42^{\prime} 13.9^{\prime \prime}$ \\
& 59701307 & 1630 & $05-07-97$ & $14^{\mathrm{h}} 24^{\mathrm{m}} 11.62^{\mathrm{s}}, 25^{\circ} 42^{\prime} 13.9^{\prime \prime}$ \\
IRC+10011 & 57701103 & 2796 & $15-06-97$ & $01^{\mathrm{h}} 06^{\mathrm{m}} 25.96^{\mathrm{s}}, 12^{\circ} 35^{\prime} 53.2^{\prime \prime}$ \\
& 57700513 & 1910 & $15-06-97$ & $01^{\mathrm{h}} 06^{\mathrm{m}} 25.96^{\mathrm{s}}, 12^{\circ} 35^{\prime} 53.2^{\prime \prime}$ \\
R Cas & 56801440 & 2204 & $06-06-97$ & $23^{\mathrm{h}} 58^{\mathrm{m}} 24.38^{\mathrm{s}}, 51^{\circ} 23^{\prime} 18.1^{\prime \prime}$ \\
\hline
\end{tabular}

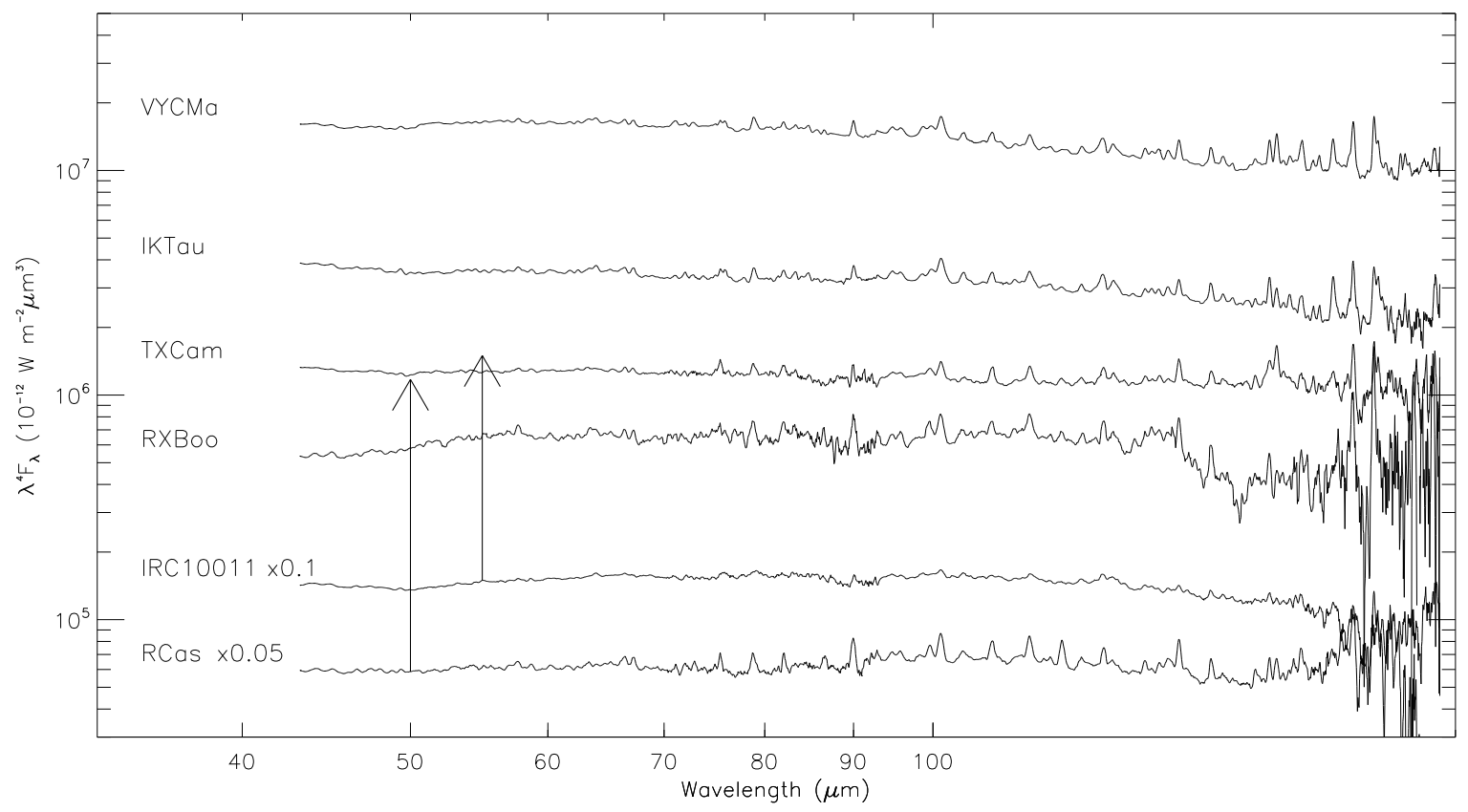

Fig. 1. LWS spectra $(45-197 \mu \mathrm{m})$ of the stars used in this paper in units of $\lambda^{4} f_{\lambda}$. From top to bottom: VY CMa, IK Tau, TX Cam, RX Boo, IRC $+10011(\times 0.1)$ and R Cas $(\times 0.05)$. The spectra of IRC+10011 and R Cas have been multiplied by factors of 0.1 and 0.05 , respectively for clarity in the plot. The arrows indicate the true flux level for these stars before the multiplication factor was applied. Each spectrum was smoothed to reduce the noise before plotting.

figure, the spectra have been smoothed with a window of 5 wavelength bins.

\section{Results: VY CMa}

In this paper we concentrate on identifying and characterising the spectral lines in the LWS data between 45 and $196 \mu \mathrm{m}$. The following sections present the line identifications for VY CMa in this range.

\subsection{Water}

The SWS spectrum of VY CMa has already been shown to be rich in the rotational lines of water (Neufeld et al. 1999). They identified at least 41 features due to water in the spectral range $29.5-45 \mu \mathrm{m}$, with transitions up to $2939 \mathrm{~K}$ above the ground state. Many of the remaining weak features in the SWS spectrum can also be explained by including higher energy transitions of water. Here, we have analysed the LWS spectrum and carefully checked all of the prominent emission features. This shows that most of the detected lines can be attributed to rotational transitions in the vibrational ground state of water.
The LWS spectra after continuum subtraction are shown in Fig. 2. The LWS spectral resolution is lower than achieved with the SWS, and many of the features are blended together. However, at longer wavelengths there are at least some gaps with few or no water lines in at all, giving the possibility to uniquely identify several other molecular species. The continuum level was estimated individually for each detector by fitting a 3rd order polynomial through the lowest points in the spectrum as described in Sect. 2. For wavelengths greater than $\sim 80 \mu \mathrm{m}$, there are sufficient gaps between emission features, and this method should give a reasonable estimate of the continuum. However, severe line blending occurs $<60 \mu \mathrm{m}$, and in this region the continuum level may have been overestimated because there is insufficiently clear space between the lines. This means that the line fluxes may be systematically underestimated. However, the fluxes for lines at wavelengths $<60 \mu \mathrm{m}$ are unlikely to be underestimated by more than a factor of a few, because sufficient contamination of the continuum would require very strong emission from lines lying $>2000 \mathrm{~K}$ above the ground state and corresponding emission $>80 \mu \mathrm{m}$ is not seen.

In Fig. 2, all the central wavelengths of all rotational orthoand para- water transitions in its vibrational ground state are marked for energies up to $2000 \mathrm{~K}$. Virtually every one of these 

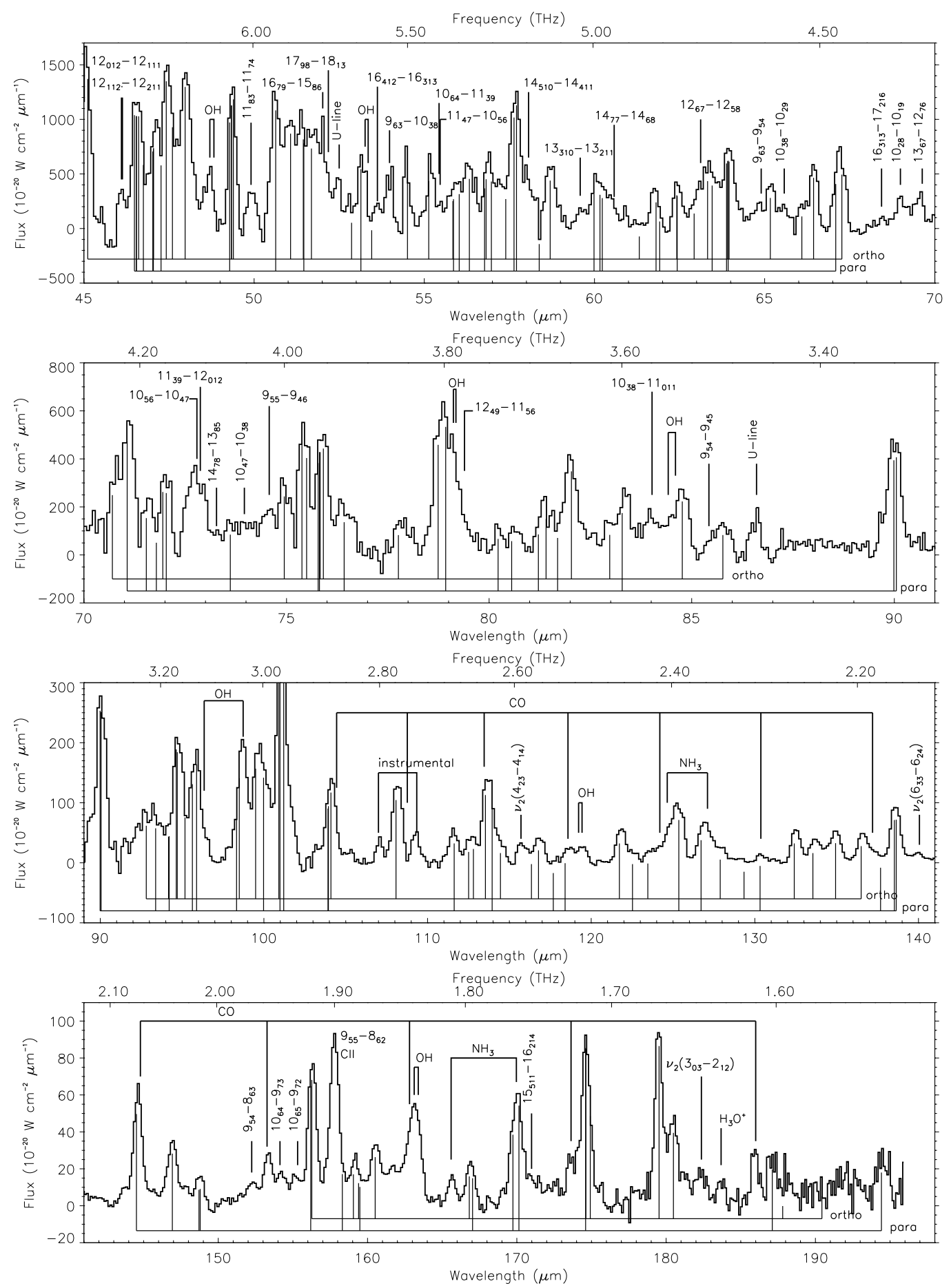

Fig. 2. Continuum subtracted LWS spectrum of VY CMa with all water line transitions with upper levels up to $2000 \mathrm{~K}$ above the ground state marked below the spectrum (para: lower, ortho: upper of the two groups) and other lines marked individually above the spectrum.

transitions is matched by a feature in the data. This upper state energy is not a fundamental limit on the features detected in the spectrum, but adopted to ease of viewing the labels displayed at the LWS spectral resolution. The inclusion of lines with higher upper state energy levels suggests that many of the low lying lines are probably blended with other water features. Several spectral regions that are free of lines from transitions with energies below $2000 \mathrm{~K}$ clearly show that higher energy transitions are present. These wavelengths are individually marked in Fig. 2 (particularly $68-70 \mu \mathrm{m}$ and $152-156 \mu \mathrm{m}$ ).

\subsection{Fitting the water lines}

In this section, we describe fitting the water lines in the vibrational ground state. Due to the large number of blended lines in the spectrum, any determination of the flux of individual lines is likely to be rather uncertain. In addition, there will be systematic errors introduced in the line fluxes due to the uncertainty in the underlying continuum level at the shortest wavelengths. In order to estimate the line fluxes, we have used a method that simultaneously fits the water lines in the vibrational ground state up to a certain energy level (upper state energy less than $2000 \mathrm{~K}$ 


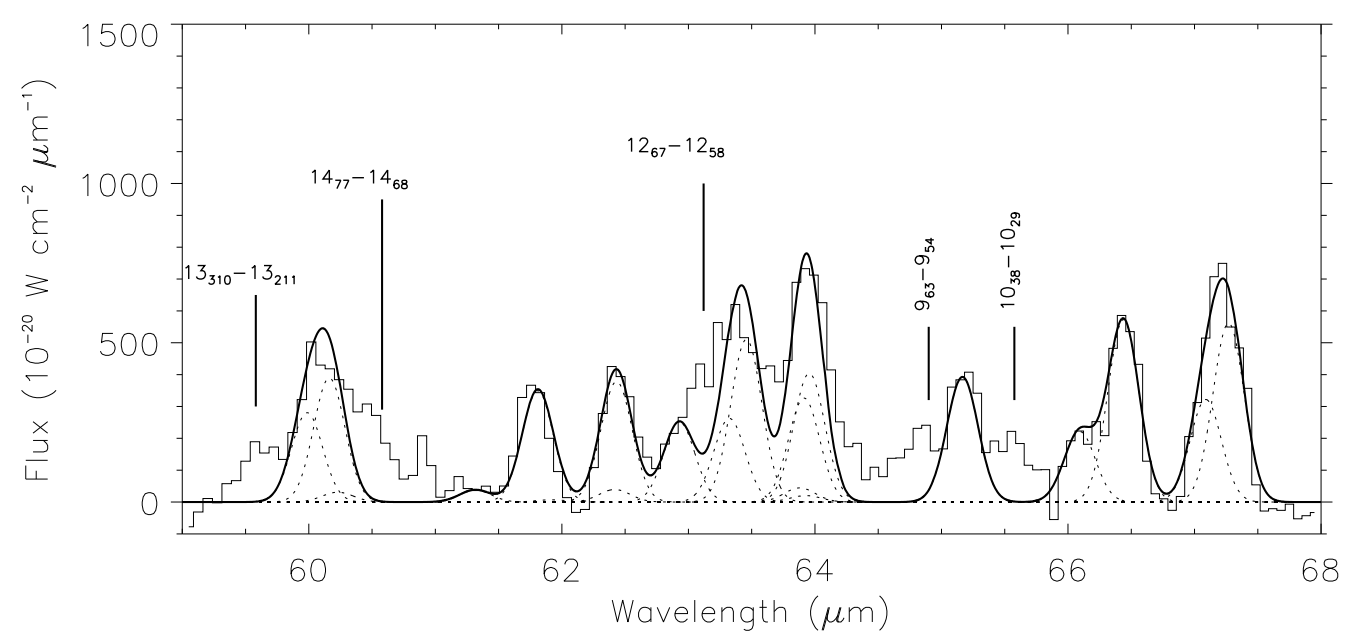

Fig. 3. An example of our water line fit for VY CMa for the region 59-68 $\mu \mathrm{m}$. The final fit is shown by the solid black line, and individual water lines by the dashed line. Additional transitions above $2000 \mathrm{~K}$ in energy are marked.

above ground). The best fit is found by increasing the flux density at each line centre until the model reaches the data. The line wavelengths are fixed at their catalogue values (taken from the JPL line catalogue; Pickett et al. 1998), and line widths fixed at the spectral resolution of the ISO detectors $(0.29 \mu \mathrm{m}$ for LWS detectors SW1-SW5 and $0.6 \mu \mathrm{m}$ for detectors LW1-LW5; Gry et al. 2003). Lines occuring within the wavelength range of each of the 10 LWS detectors are fitted together.

The flux densities at the centres of all lines measured by the detector are increased by a step that is weighted (for each line) relative to the Einstein coefficient for spontaneous emission, $A_{u l}$, (with values taken from the JPL catalogue; Pickett et al. 1998), and $\exp \left(-E_{u} / k T_{\text {weight }}\right)$, where $E_{u} / k$ is the energy of the upper state above ground in $K$, and $T_{\text {weight }}$ is a single temperature applied to all lines. This ensures that lines with higher weight are given preference in blends. However, it does not necessarily mean that the final flux will be distributed following the weights - the actual shape of the data may allow a line with a lower weight to have a higher flux than one with a high weight. The weighting scheme does not treat ortho and para lines separately. The basic step size in the model is set to 0.01 times flux density at the wavelength of the strongest line, and the individual step for all other lines are multiplied by their weights,

$I_{\lambda-\text { model }}^{\prime}=I_{\lambda-\text { model }}+w_{\lambda} S$

where $I_{\lambda}$ is the flux density at the centre of the line, $w_{\lambda}$ is the weight for that line, defined by,

$w_{\lambda}=\frac{A_{u l}}{\operatorname{MAX}\left[A_{u l}\right]} \frac{\exp \left(-E_{u} / k T_{\text {weight }}\right)}{\operatorname{MAX}\left[\exp \left(-E_{u} / k T_{\text {weight }}\right)\right]}$

and $s$ is the basic step size, defined by,

$s=\frac{\operatorname{MAX}\left[I_{\lambda \text {-data }}\right]}{100}$

where the operation MAX finds the maximum value over the lines occuring within the wavelength range of the particular LWS detector being fitted.

A first estimate for the value of $T_{\text {weight }}$ to use in this weighting function was determined from a rotation diagram (see Sect. 3.3) using a fit weighted relative to $A_{u l}$ only,

$w_{\lambda}=\frac{A_{u l}}{\operatorname{MAX}\left[A_{u l}\right]}$.
Figure 3 shows a fairly representative example of the final fit, for the region 59-68 $\mu \mathrm{m}$. This region clearly shows several higher energy lines that were not included in the fit.

The advantage of this technique to estimate the line fluxes is that it is much faster than carrying out a multiple Gaussian $\chi^{2}$ fit. Moreover, the flux in complicated blends is not overestimated (as it could be in a free Gaussian fit if the line shape is affected by other species). The disadvantage is that it takes no account of the overall excitation of the molecule, except within blends where the lines are explicitly weighted according to $A_{u l}$ and $T_{\text {weight }}$. The final errors on each line flux are highly correlated in the various blended groups and therefore difficult to rigorously estimate. However, this fitting method provides a good starting point to investigate the spectrum, and represents the best that can be achieved using data observed with the available spectral resolution.

In order to check the reliability of the automatic fitting routine, we compared the fit using several different weighting schemes: no weight, weighted relative to $A_{u l}$ only (Eq. (4)), and weighted relative to $A_{u l}$ and $\exp \left(-E_{u} / k T_{\text {weight }}\right.$ ) (Eq. (2)). For the few completely unblended lines, we also carried out a manual fit using ISAP and in general this agrees within the errors with the automatic procedure. The unblended line fluxes for these different fit methods are shown for VY CMa and IK Tau in Table 4. We estimate that the fitting errors for unblended lines are 15-20\%.

The final line fluxes determined from the fit for all lines up to $2000 \mathrm{~K}$ above the ground state (including blends) are shown in Table A.1, for all of the stars investigated. In order to check the blended fits, we have compared our results for R Cas with those obtained by Truong-Bach et al. (1999). Our fitted values are in reasonable agreement (in a line by line comparison, many fluxes agree to better than $50 \%$, and the remaining discrepencies can be explained by the fact that we have included more blends). We estimate that our blended line fluxes are probably of the correct order of magnitude (unless the assumptions used for the weighting scheme within the fit are wildly incorrect).

We have also checked our fitted fluxes with the analysis of Maercker et al. (2008) who fitted water lines in a similar sample of stars. In general at long wavelengths where the lines are well separated, we find very good agreement in fitted fluxes. However, the difference in our analysis is that we have included the possibility of blends with higher energy lines. This is clearly necessary in VY CMa to account for all of the detected features. 
In the analysis of Maercker et al. (2008), they sometimes calculated model values lower than their quoted line fits to the data. This may be partly due to not identifying blends - some of our fitted fluxes which include blends (where Maercker et al. did not include a blend) give values closer to their model predictions. On the other hand, in some cases, our simple treatment that includes many blended lines seems to underestimate the flux compared to their model, suggesting that we may have not distributed the flux correctly. However, this comparison shows that in these stars, it is very important to consider that detected features may be due to blends of water transitions over a very wide range in energy, and it is not enough to merely consider the (strongest) lower level lines.

\subsection{Excitation of $\mathrm{H}_{2} \mathrm{O}$}

In order to investigate the fitted line fluxes, we have used a simple rotation diagram approach (e.g. Goldsmith \& Langer 1999). Assuming that all transitions are in local thermodynamic equilibrium (LTE), there is a linear relationship between the natural logarithm of the upper state column density per statistical weight and the energy above the ground state (i.e. following the Boltzmann distribution),

$\ln \left(\frac{N_{u}}{g_{u}}\right)=\ln \left(\frac{N_{\text {tot }}}{Q}\right)-\frac{1}{T} \frac{E_{u}}{k}$

where $N_{u}$ is the column density in the upper state, $g_{u}$ is the statistical weight of the upper state, $N_{\text {tot }}$ is the total column density, and $Q$ is the partition function at temperature $T$. A plot of $\ln \left(N_{u} / g_{u}\right)$ versus $E_{u} / k$ can be fitted with a straight line with gradient $1 / T$ and intercept $\ln \left(N_{\text {tot }} / Q\right)$. We have calculated the column densities assuming optically thin emission lines using the relationship,

$N_{u}=\frac{4 \pi \lambda}{h c A_{u l} \Omega} \int I_{\lambda-\text { data }} \mathrm{d} \lambda$

where $\Omega$ is the solid angle subtended by the water-emitting region around the star (assumed to be a disk with diameter $4^{\prime \prime}$ ) and $\int I_{\lambda \text {-data }} \mathrm{d} \lambda$ is the measured flux. This choice of the source size is motivated by the size of the $\mathrm{CO}$ emission region imaged by Muller et al. (2007). Our adoption of a uniform temperature across a region of this size is highly unrealistic and so our results should be taken as qualitative estimates (also see further caveats on the results below).

Figure 4 shows the rotation diagram for VY CMa. Even though we know that this model does not accurately describe reality (VY CMa actually has a very complex environment - see Sect. 5), and many of the lines are likely to be optically thick and/or subthermally excited, we think it provides an instructive starting point to view the fitted fluxes, particularly since the number of lines that we are fitting is very large (141 lines used in the fit). The lower energy lines in Fig. $4(<1500 \mathrm{~K})$ are unlikely to be completely optically thin, and Maercker et al. (2008) found that the water lines in their sample of stars were generally subthermally excited (although more thermalised in IK Tau than the other stars they looked at).

Although most of the results cluster along a straight line, there is a large scatter. This is exactly the effect we should expect to see for a nonlinear molecule which has a mixture of optically thick and thin lines that are not necessarily in LTE (Goldsmith \& Langer 1999, see their Figs. 5 and 8). The lines least likely to be optically thick are those with upper state energies above $1500 \mathrm{~K}$,

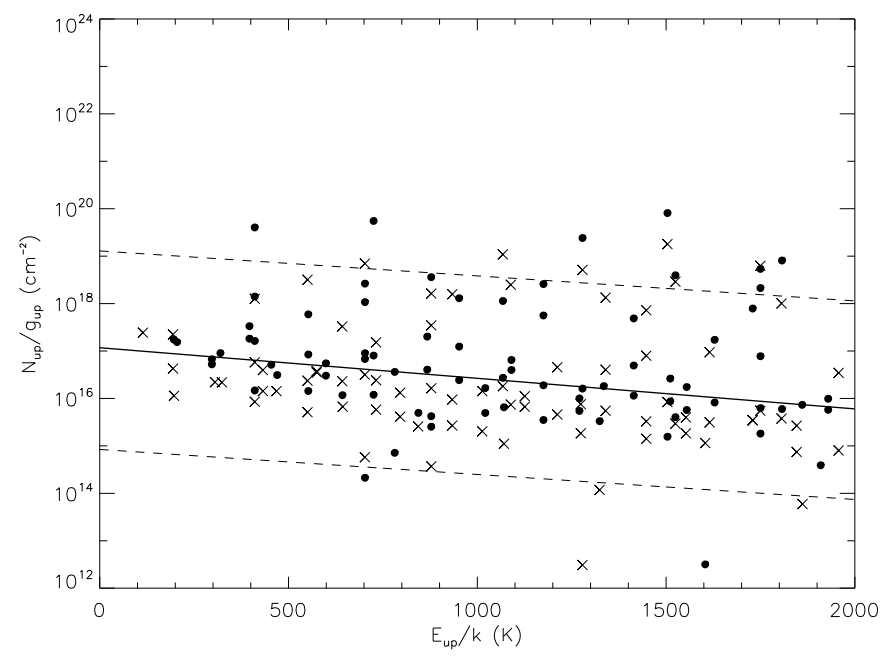

Fig. 4. Rotation diagram resulting from fit to water lines in the LWS spectrum of VY CMa weighted by $A_{u l}$ and $E_{u}$. Ortho water lines are shown as filled circles and para water lines as crosses. The best fit to lines with upper state energies less than $2000 \mathrm{~K}$ (ignoring outlying points) is shown, giving a rotational temperature of $670_{-130}^{+210} \mathrm{~K}$.

although these lines are more likely to be blended, and so have large fitting errors.

Taking into account the above uncertainties, the fitted value for the total column density should be taken as a lower limit, and the temperature as a very rough estimate of the actual kinetic temperature (also bearing in mind that we expect a range of different thermal environments). The best fit straight line has a slope corresponding to a rotational temperature of $T_{\text {rot }}=$ $670_{-130}^{+210} \mathrm{~K}$ (the error was derived only taking into account the scatter in the data points). There does not appear to be a significant difference between the ortho- and para- lines. Due to the challenges described earlier regarding accurate flux estimation, we have discounted far outlying points by carrying out a $2 \sigma$ clip. These points, which can lie far above the best fit line, could be due to miss-fitting the flux of lines in blends. The rest of the scatter is due to real departures from the simple model as described above. More detailed modelling, beyond the scope of this paper is required to investigate these effects. The isotopic lines of $\mathrm{H}_{2}^{18} \mathrm{O}$ could be used to investigate the optical depths, but this will require future higher resolution observations (a useful limit on the $\mathrm{H}_{2}^{18} \mathrm{O}$ emission is difficult with the current LWS data).

The best fit intercept point gives $N_{\text {tot }} / Q=(1.17 \pm 0.2) \times$ $10^{17} \mathrm{~cm}^{-2}$. The partition function at $300 \mathrm{~K}$ is given in the JPL catalogue (Pickett et al. 1998) as 178.12. The value scales as $T^{1.5}$ which leads to a partition function of 594.47 at $670 \mathrm{~K}$, and this gives a lower limit on the total water column density of $(7.0 \pm$ 1.2) $\times 10^{19} \mathrm{~cm}^{-2}$.

\subsection{Vibrationally excited water}

In addition to transitions from the vibrational ground state of water, Neufeld et al. (1999) also identified 4 features with water in its vibrationally excited $v_{2}=1$ state. Rotational transitions of water in its $v_{2}=1$ state have also been observed at millimetre and submillimetre wavelengths, showing that the emission probably comes from a region close to the star (Menten \& Melnick 1989; Menten et al. 2006). At the spectral resolution of the LWS grating, vibrationally excited water is much harder to separate. However, in the longer wavelength detectors there are three features that match with wavelengths of $v_{2}=1$ 
Table 3. Possible rotational lines from the vibrationally excited $v_{2}=1$ state of water in the LWS spectrum of VY CMa.

\begin{tabular}{cccc}
\hline \hline Transition & $\begin{array}{c}\text { Wavelength } \\
(\mu \mathrm{m})\end{array}$ & $\begin{array}{c}\text { Energy of } \\
\text { upper state }(\mathrm{K})\end{array}$ & $\begin{array}{c}\text { Flux }^{a} \\
\left(10^{-19} \mathrm{~W} \mathrm{~cm}^{-2}\right)\end{array}$ \\
\hline $3_{03}-2_{12}$ & 182.36 & 2491.8 & $1.3 \pm 0.3$ \\
$6_{33}-6_{24}$ & 140.06 & 3284.2 & $1.2 \pm 0.2$ \\
$4_{23}-4_{14}$ & 115.71 & 2745.2 & $2.3 \pm 0.8$ \\
\hline
\end{tabular}

Notes. ${ }^{(a)}$ Errors do not include systematic uncertainty in the baseline subtraction $(\sim 30 \%)$.

rotational transitions. These are shown in Table 3. It is possible that other vibrationally excited lines exist in the spectrum but all other transitions with similar energies occur in blends. Also, the ground state transitions were identified first, which may mean that some vibrationally excited lines could have been misidentified as ground state ones (for example, the $v_{2}=122_{12}-1_{01}$ line occurs at $170.928 \mu \mathrm{m}$, close to the feature we have labelled as $15_{511}-16_{214}$ in Fig. 2). Higher spectral resolution observations would be required to unambiguously identify all of the vibrationally excited lines.

Menten et al. (2006) detected two transitions of vibrationally excited water using the $12 \mathrm{~m}$ Atacama Pathfinder Experiment telescope (APEX): $5_{23}-6_{16}$ at $336.2 \mathrm{GHz}$ and $6_{61}-7_{52}$ at 293.6 GHz. They used a model to calculate the optical depths and intensities of their measured lines, assuming that the transitions were thermalised. We have applied the same model to calculate the optical depth and predicted flux of our three detected FIR transitions. The line wavelengths and transition parameters were taken from the JPL line catalogue (Pickett et al. 1998). If we make the same assumptions about the emitting region ( $T=1000 \mathrm{~K}$, thermalised lines, uniform region within $0.05^{\prime \prime}$ from the star) and assume a line width of $20 \mathrm{~km} \mathrm{~s}^{-1}$ (the same as the APEX lines), we derive high optical depths of 500, 270 and 600 for the three lines in Table 3 . The predicted fluxes are a few $10^{-21} \mathrm{~W} \mathrm{~cm}^{-2}$, which underestimate our measured LWS fluxes by factors of 36-86.

The model predictions were closer for the sub-millimetre lines observed by Menten et al. (2006), underestimating their measured intensities by only a factor of 2.3 and 5 , and a factor of 3 for the $5_{50}-6_{43} 232.6 \mathrm{GHz}$ transition observed by Menten $\&$ Melnick (1989). The underestimated intensity was explained by the fact that the $294 \mathrm{GHz}$ line may be boosted by weak maser action.

We have also applied the model to the SWS lines observed by Neufeld et al. (1999), predicting that the SWS lines should be stronger than the LWS lines, which is not observed. The SWS fluxes measured by Neufeld et al. (1999) are reproduced by the model within factors of 2-8. The optical depths for the SWS lines are predicted to be in the order of $10^{4}$.

The model seems to be much worse at predicting the FIR fluxes than those in the sub-millimetre and mid-infrared. At the high optical depths predicted, the only parameters in the model that affect the final fluxes are the temperature of the emitting region, the line width and assumed source size. The flux calculated by the model could be brought into better agreement by a combination of increasing the temperature and size of the emitting region, as the line fluxes are $\sim$ linearly proportional to both. However, increasing the assumed emission region size has a large effect on the predicted sub-mm main beam brightness temperatures. The excitation temperature does not have a large impact on the sub-mm lines, but a much higher temperature would be needed than is indicated by previous measurements (see Menten et al. 2006).

This either indicates that there is another problem with the simple model (for example, different excitation conditions for the FIR lines) or that the measured LWS fluxes are overestimated (possibly due to additional line blending which has not been taken into account here). Observations at higher spectral resolution are needed to confirm the fluxes, and more detailed modelling is required, taking into account both the sub-mm and infrared lines.

\subsection{CO lines}

In the LWS spectrum of VY CMa, the water line density decreases toward the longer wavelengths. $\mathrm{CO}$ is known to be an abundant molecule in O-rich circumstellar envelopes and many observations have been made via its millimetre and submillimetre transitions (e.g. Kemper et al. 2003; Decin et al. 2006; Ziurys et al. 2009). The lowest energy CO transition in the ISO spectral range is $J=14-13$, with upper state energy of $580 \mathrm{~K}$ above the ground state.

Several CO lines have previously been reported in the LWS spectral range toward R Cas (Truong-Bach et al. 1999) and W Hya (Barlow et al. 1996). In addition gaseous CO absorption has been observed around $4.5 \mu \mathrm{m}$ in the SWS spectra of O-rich stars (e.g. Sylvester et al. 1997).

In the LWS spectrum of VY CMa, we observe some contribution to the spectrum from all CO lines from $J=14-13$ up to $J=25-24$. These lines are labeled in Fig. 2. The higher energy lines are difficult to identify due to blending with water lines and the best detection is of the lowest energy lines which are generally well separated. The fluxes of these lines at long wavelengths were determined in two ways and are shown in Table 5. As a first estimate, the $\mathrm{CO}$ fluxes were determined by fitting the excess emission after subtracting the water line fit described in Sect. 3.2. The flux was also determined by a manual Gaussian fit using ISAP, taking account of nearby blended lines with a multiGaussian fit. The automatic fit generally underestimates the flux compared to the manual fit, probably because water was given preference in the blends for the automatic fit (although the automatic fit is within the errors of the ISAP fit). The true flux is probably between the two values.

We have carried out a rotation diagram analysis of the $\mathrm{CO}$ fluxes in the same way as described in Sect. 3.3 for $\mathrm{H}_{2} \mathrm{O}$. This is shown in Fig. 5, with a fit to the 4 lowest energy lines (the fit ignores the fluxes for $J=19-18$ and $J=21-20$ which have very large error bars). This gives a rough estimate of the rotational temperature and total column density as we are only fitting a few lines. However, the results should be more reliable than for $\mathrm{H}_{2} \mathrm{O}$, because the high excitation $\mathrm{CO}$ lines are likely to be optically thin. We have used the manual ISAP fluxes from Table 5 and fitted a straight line weighted by their errors. This gives $T_{\text {rot }}=260 \pm 140 \mathrm{~K}$ and $N_{\text {tot }} / Q=\left(8_{-6}^{+22}\right) \times 10^{15} \mathrm{~cm}^{-2}$. The partition function at $300 \mathrm{~K}$ is given in the JPL catalogue (Pickett et al. 1998) as 108.865, and this leads to a total CO column density of $N_{\text {tot }}=\left(9_{-7}^{+24}\right) \times 10^{17} \mathrm{~cm}^{-2}$.

This column density is approximately 100 times lower than the $\mathrm{H}_{2} \mathrm{O}$ column density calculated in Sect. 3.3, showing that generally in the VY CMa envelope, water is the dominant oxygen carrier (although the $\mathrm{H}_{2} \mathrm{O}$ and $\mathrm{CO}$ come from different temperature regions). 
A\&A 510, A80 (2010)

Table 4. Fitted fluxes for the unblended lines in the spectrum of VY CMa and IK Tau.

\begin{tabular}{|c|c|c|c|c|c|}
\hline Transition & $\begin{array}{l}\text { Wavelength } \\
\qquad(\mu \mathrm{m})\end{array}$ & $\begin{array}{c}\text { No weight } \\
\text { fit flux } \\
\left(10^{-19} \mathrm{~W} \mathrm{~cm}^{-2}\right)\end{array}$ & $\begin{array}{c}\text { Weighted } \\
\text { fit flux } \\
\left(10^{-19} \mathrm{~W} \mathrm{~cm}^{-2}\right)\end{array}$ & $\begin{array}{c}\text { Manual } \\
\text { fit flux } \\
\left(10^{-19} \mathrm{~W} \mathrm{~cm}^{-2}\right)\end{array}$ & $\begin{array}{c}\text { IK Tau weighted } \\
\text { fit flux } \\
\left(10^{-19} \mathrm{~W} \mathrm{~cm}^{-2}\right)\end{array}$ \\
\hline $4_{32}-3_{21}$ & 58.699 & 15.5 & 15.1 & $27.8 \pm 5.4$ & 3.3 \\
\hline $6_{16}-5_{05}$ & 82.031 & 10.9 & 11.0 & $14.0 \pm 2$ & 2.5 \\
\hline $2_{21}-1_{10}$ & 108.073 & 7.2 & 7.1 & $9.2 \pm 1.1$ & 1.9 \\
\hline $4_{32}-4_{23}$ & 121.722 & 3.1 & 3.0 & $3.8 \pm 1.0$ & 0.73 \\
\hline $4_{23}-4_{14}$ & 132.408 & 3.1 & 3.0 & $3.6 \pm 0.8$ & 0.49 \\
\hline $8_{36}-7_{43}$ & 133.549 & 2.2 & 2.1 & $2.7 \pm 0.8$ & 0.54 \\
\hline $5_{14}-5_{05}$ & 134.935 & 3.1 & 3.0 & $3.8 \pm 0.8$ & 0.58 \\
\hline $3_{30}-3_{21}$ & 136.496 & 2.9 & 2.8 & $3.3 \pm 0.9$ & 0.73 \\
\hline $4_{31}-4_{22}$ & 146.923 & 2.0 & 2.0 & $2.5 \pm 0.7$ & 0.29 \\
\hline $5_{32}-5_{23}$ & 160.510 & 2.0 & 1.9 & $2.5 \pm 0.4$ & 0.55 \\
\hline $2_{12}-1_{01}$ & 179.527 & 5.3 & 5.3 & $6.3 \pm 0.7$ & 1.1 \\
\hline $2_{21}-2_{12}$ & 180.488 & 2.6 & 2.6 & $3.4 \pm 0.4$ & 0.85 \\
\hline $6_{33}-5_{42}$ & 194.422 & 1.5 & 1.5 & $1.8 \pm 0.3$ & 0.44 \\
\hline
\end{tabular}

Notes. ${ }^{(a)}$ Errors do not include systematic uncertainty in the baseline subtraction $(\sim 30 \%)$.

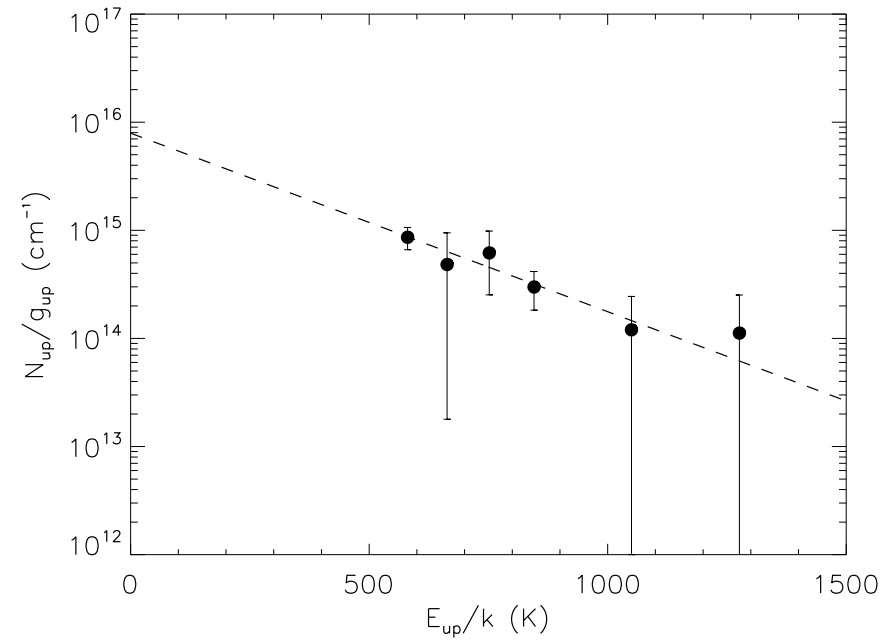

Fig. 5. Rotation diagram for the CO lines observed towards VY CMa. The dashed line is a fit to the 4 lowest energy lines using the manually fitted fluxes from Table 5 .

Table 5. Fitted fluxes for the CO lines observed above $120 \mu \mathrm{m}$ for VY CMa.

\begin{tabular}{cccc}
\hline \hline Transition & $\begin{array}{c}\text { Wavelength } \\
(\mu \mathrm{m})\end{array}$ & $\begin{array}{c}\text { Auto } \\
\text { fit flux } \\
\left(10^{-19} \mathrm{~W} \mathrm{~cm}^{-2}\right)\end{array}$ & $\begin{array}{c}\text { Manual } \\
\text { fit flux }^{a} \\
\left(10^{-19} \mathrm{~W} \mathrm{~cm}^{-2}\right)\end{array}$ \\
\hline$J=14-13$ & 185.999 & 1.72 & $1.96 \pm 0.4$ \\
$J=15-14$ & 173.631 & 1.35 & $1.88 \pm 1.3$ \\
$J=16-15$ & 162.812 & 2.37 & $2.69 \pm 1.4$ \\
$J=17-16$ & 153.267 & 1.54 & $2.00 \pm 0.6$ \\
$J=18-17$ & 144.784 & - -Blend- & - Blend- \\
$J=19-18$ & 137.196 & 1.06 & $1.31 \pm 1.1$ \\
$J=20-19$ & 130.369 & - -Blend- & - Blend- \\
$J=21-20$ & 124.193 & 1.60 & $0.92 \pm 2.0$ \\
\hline
\end{tabular}

Notes. ${ }^{(a)}$ Errors do not include systematic uncertainty in the baseline subtraction $(\sim 30 \%)$.

\subsection{Other lines: $\mathrm{OH}, \mathrm{NH}_{3}$ and $\mathrm{H}_{3} \mathrm{O}^{+}$}

Several other lines were detected in the spectrum of VY CMa. These are summarised in Table 6 and described in the following paragraphs.
We detect strong emission from $\mathrm{OH}$ at $163 \mu \mathrm{m}$, which is blended with the CO $J=16-15$ line. This line is due to the lowest energy pure rotational transition in the ${ }^{2} \Pi_{1 / 2}$ ladder of $\mathrm{OH}$. Some contribution from the next transition in this ladder at $99 \mu \mathrm{m}$ is probably also detected. In addition we detect emission in several other transitions of $\mathrm{OH}$ such as the lowest rotational line in the ${ }^{2} \Pi_{3 / 2}$ ladder at $119 \mu \mathrm{m}$. As the cross ladder transition at $34 \mu \mathrm{m}$ is seen in absorption in the SWS spectrum (Neufeld et al. 1999), we might also expect the other cross ladder transitions in the LWS range to be in absorption (e.g. the other transitions from the ${ }^{2} \Pi_{3 / 2} J=3 / 2$ level at $53 \mu \mathrm{m}$ and $79 \mu \mathrm{m}$ ). However, these are both blended with water emission.

The FIR lines of ammonia in its $v_{2}$ bending-inversion mode are rotation-inversion transitions, with the lowest energy transition $K=2, J=3-2$ at 165.60 and $169.97 \mu \mathrm{m}$. There is a clear emission peak at $165.60 \mu \mathrm{m}$ in the spectrum of VY CMa (see Fig. 2). This does not overlap with any water lines and has a width comparable to the instrument resolution. We think that the assignment of this feature to $\mathrm{NH}_{3}$ is secure because it does not appear to be blended. There are only a few other species that could provide alternative explanations for the line: the most likely two choices are $\mathrm{HNC} J=20-19$ and $\mathrm{H}_{3} \mathrm{O}^{+} K=0, J=5-$ 4. The HNC line has a high energy upper level at $913 \mathrm{~K}$ and the corresponding transition in $\mathrm{HCN}$ at $161.35 \mu \mathrm{m}$ is not clearly detected. If the line was due to $\mathrm{H}_{3} \mathrm{O}^{+}$, there should have been other features present which are not observed. The line is also observed in the spectrum of IK Tau (see Sect. 4).

The $\mathrm{NH}_{3}$ line at $169.97 \mu \mathrm{m}$ occurs in a blend with water lines. The transition at the next highest energy in $\mathrm{NH}_{3}$ is $K=3$, $J=4-3$ at 124.65 and $127.11 \mu \mathrm{m}$. Both these lines occur in the wing of water emission (but are marked in Fig. 2).

Ammonia has been observed in the envelopes of both carbon and oxygen rich stars - the first detection in an O-rich envelope was made by McLaren \& Betz (1980) in VY CMa, VX Sgr and IRC +10420 . They observed several vibration-rotation transitions in the $\gamma_{2}$ band at 10.5 and $10.7 \mu \mathrm{m}$. Subsequently these transitions have been measured in more detail for VY CMa by Monnier et al. (2000) and references therein. These observations show that $\mathrm{NH}_{3}$ probably forms near the termination of the gas acceleration phase.

The only detection of the radio inversion transitions of ammonia in O-rich stars was made by Menten \& Alcolea (1995) toward IK Tau and IRC+10420. They found that the strongest 
Table 6. Summary of other lines detected in the VY CMa spectrum.

\begin{tabular}{lcc}
\hline \hline Transition & $\begin{array}{c}\text { Wavelength } \\
(\mu \mathrm{m})\end{array}$ & Comment \\
\hline $\mathrm{OH}^{2} \Pi_{1 / 2} J=3 / 2-1 / 2$ & $163.1 / 163.4$ & blend with $\mathrm{CO}$ \\
$\mathrm{OH}^{2} \Pi_{3 / 2} J=5 / 2-3 / 2$ & $119.2 / 119.4$ & well separated \\
$\mathrm{NH}_{3} K=2, J=3^{+}-2^{-}$ & 165.60 & well separated \\
$\mathrm{NH}_{3} K=2, J=3^{-}-2^{+}$ & 169.97 & blend with $\mathrm{H}_{2} \mathrm{O}$ \\
$\mathrm{H}_{3} \mathrm{O}^{+} K=1, J=2-2$ & 183.68 & possible identification \\
$\mathrm{C}^{+}{ }^{2} \mathrm{P}_{3 / 2}-{ }^{2} \mathrm{P}_{1 / 2}$ & 157.74 & blend with $\mathrm{H}_{2} \mathrm{O}$ \\
$\mathrm{U}-$-line & 52.5 & \\
U-line & 86.6 & \\
\hline
\end{tabular}

inversion transition for IK Tau was $K=3, J=3-3$ whereas for IRC +10420 was $K=1, J=1-1$. This implies that with a higher mass loss rate, photodissociation occurs further from the star and the $\mathrm{NH}_{3}$ emission comes from a lower temperature region.

The $\mathrm{H}_{3} \mathrm{O}^{+}$ion has a similar pyramidal structure to the isoelectronic molecule $\mathrm{NH}_{3}$. In an analogous way to $\mathrm{NH}_{3}$, the oxygen atom can tunnel through the plane of the molecule leading to an inversion splitting of the rotational levels. However, for $\mathrm{H}_{3} \mathrm{O}^{+}$, the inversion splitting is very large $\left(55.34 \mathrm{~cm}^{-1}\right.$ for the $v_{2}$ bending-inversion mode; Liu et al. 1986). This means that the pure inversion transitions occur at FIR wavelengths rather than in the radio regime as for ammonia. Several rotationinversion transitions at $1 \mathrm{~mm}$ have previously been detected in giant molecular clouds (Wootten et al. 1991; Phillips et al. 1992) and the lines near $300 \mathrm{GHz}$ mapped toward Sgr B2 (van der Tak et al. 2006). Several of the FIR transitions have also been observed in absorption toward Sgr B2 with ISO (Goicoechea \& Cernicharo 2001; Polehampton et al. 2007).

The peak abundance of $\mathrm{H}_{3} \mathrm{O}^{+}$in oxygen-rich circumstellar envelopes is predicted to be $\sim 10^{-7}$ (Mamon et al. 1987), with the major formation route being the photodissociation of $\mathrm{H}_{2} \mathrm{O}$ and $\mathrm{OH}$. Excitation is probably via the absorption of mid-IR radiation rather than collisions and thus absorption bands may also be observable at 10 and $17 \mu \mathrm{m}$. The only (possible) detection of $\mathrm{H}_{3} \mathrm{O}^{+}$toward an evolved star so far is the $2 \sigma$ feature observed toward VY CMa by Phillips et al. (1992) at the correct velocity for the $K=0 J=3-2396 \mathrm{GHz}$ transition.

In the LWS grating spectrum of VY CMa, the spectral resolution is low and the inversion and rotation-inversion transitions are difficult to separate from the water lines. However, there is an unidentified feature at the correct wavelength for the $K=1$ $J=2-2$ transition at $183.68 \mu \mathrm{m}$. The other low energy inversion transition may contribute to the (water) emission features around $180-181 \mu \mathrm{m}$. The rotation-inversion transitions are harder to positively identify as they occur at shorter wavelengths where the density of water lines is higher.

In several O-rich star spectra, a feature at $157.8 \mu \mathrm{m}$ has been assigned to the atomic fine structure line of ionised carbon at $157.74 \mu \mathrm{m}$ (Truong-Bach et al. 1999; Sylvester et al. 1997). This line also appears in the VY CMa spectrum but no other atomic lines are clearly visible. Sylvester et al. (1997) attribute this line to bad subtraction of the galactic background in their sources. However, at least some contribution to it can be explained by the $9_{55}-8_{62}$ para water line at $157.88 \mu \mathrm{m}$. The upper level for this transition occurs $2031 \mathrm{~K}$ above the ground state. In VY CMa there could be some contribution from both lines.

There are several remaining unassigned features in the spectrum and these are labeled as "U-lines" in Fig. 2. In addition, there are several species which have transitions in the range and are predicted to be reasonably abundant in O-rich circumstellar envelopes, but are not possible to detect due to line blending with water (e.g. HCN, $\mathrm{H}_{2} \mathrm{~S}$; Willacy \& Millar 1997).

\section{Comparison with other stars}

The spectrum of the oxygen rich Mira variable IK Tau shows remarkable similarity to that of VY CMa. However, the line fluxes shown in Fig. 6 are $\sim 4$ times weaker and the continuum level $\sim 10$ times lower. Since it was only observed for about twice the integration time of VY CMa, the signal-to-noise of the spectrum appears lower than that of VY CMa. Within the noise level and accuracy of the continuum subtraction, all of the lines observed in the VY CMa spectrum are present in the IK Tau spectrum, generally with very similar relative intensities. Figure 6 shows the spectrum of IK Tau scaled by a factor of 4.5 and compared to VY CMa. It is clear that the same lines are detected in each object. There are, however, a few exceptions where there are marked differences: there is much weaker $\mathrm{OH}$ emission with respect to water in IK Tau. The $\mathrm{OH}$ line at $119 \mu \mathrm{m}$ is not present and there may be less contribution to the $163 \mu \mathrm{m} \mathrm{CO} / \mathrm{OH}$ blend from $\mathrm{OH}$. Also the line at $99 \mu \mathrm{m}$ is weaker in IK Tau. However, the other lines such as $\mathrm{CO}, \mathrm{NH}_{3}$ and the possible $v_{2}=1$ water lines are all present in both stars. In addition, the unidentified feature at $86 \mu \mathrm{m}$ is present in both spectra. There is only one feature that is present in IK Tau but not in VY CMa at $73.8 \mu \mathrm{m}$.

In Fig. 6, we also plot the other stars from Table 1. In order to compare the spectral lines, we have multiplied each continuum subtracted spectrum by factors of 4.5 for IK Tau and R Cas; 6 for TX Cam and 10 for RX Boo and IRC+10011. We have also smoothed the noisy spectra. Several key spectral lines are shown.

The other stars of a similar type to IK Tau (TX Cam, RX Boo, IRC+10011) have a much lower signal to noise ratio and their lines are weaker than IK Tau. However, the majority of detections in VY CMa and IK Tau also seem to be present in these stars.

We have fitted the water lines in the vibrational ground state for the other stars in the same way as for VY CMa and the results are shown in Table A.1. We have carried out a rotation diagram analysis for each star as described in Sect. 3.3, and the results are shown in Table 7 , giving a rough estimate of the rotational temperature and a lower limit for the total water column density.

The rotational temperatures for the other stars are very simi$\operatorname{lar}$ (within 25\%) to the value found for VY CMa, which indicates that the $\mathrm{H}_{2} \mathrm{O}$-emitting gas is excited under similar conditions. If the excitation is dominated by collisions, $T_{\text {rot }}$ should reflect the kinetic temperature of the gas because of the high densities of AGB star envelopes. Alternatively, radiation may dominate the excitation, in which case $T_{\text {rot }}$ would reflect the temperature of the ambient radiation field. The only star where $T_{\text {rot }}$ deviates from the trend is IRC +10011 , but in this case, the error on $T_{\text {rot }}$ is rather large (due to a larger scatter in the data points in the rotation diagram).

The derived $\mathrm{H}_{2} \mathrm{O}$ column densities for the other stars are lower than for VY CMa, with values ranging from $2 \%$ to $12 \%$ of the value for VY CMa. Since the other stars also have 10100 times lower mass loss rates (see Table 1), this result indicates that water is also the main oxygen carrier in the other star

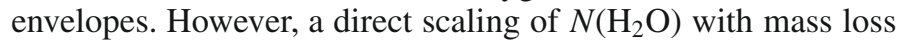
rate does not appear: the ratio of $\mathrm{H}_{2} \mathrm{O}$ column density and mass loss rate shows a large variation over our sample of stars (factor of $>10)$. 


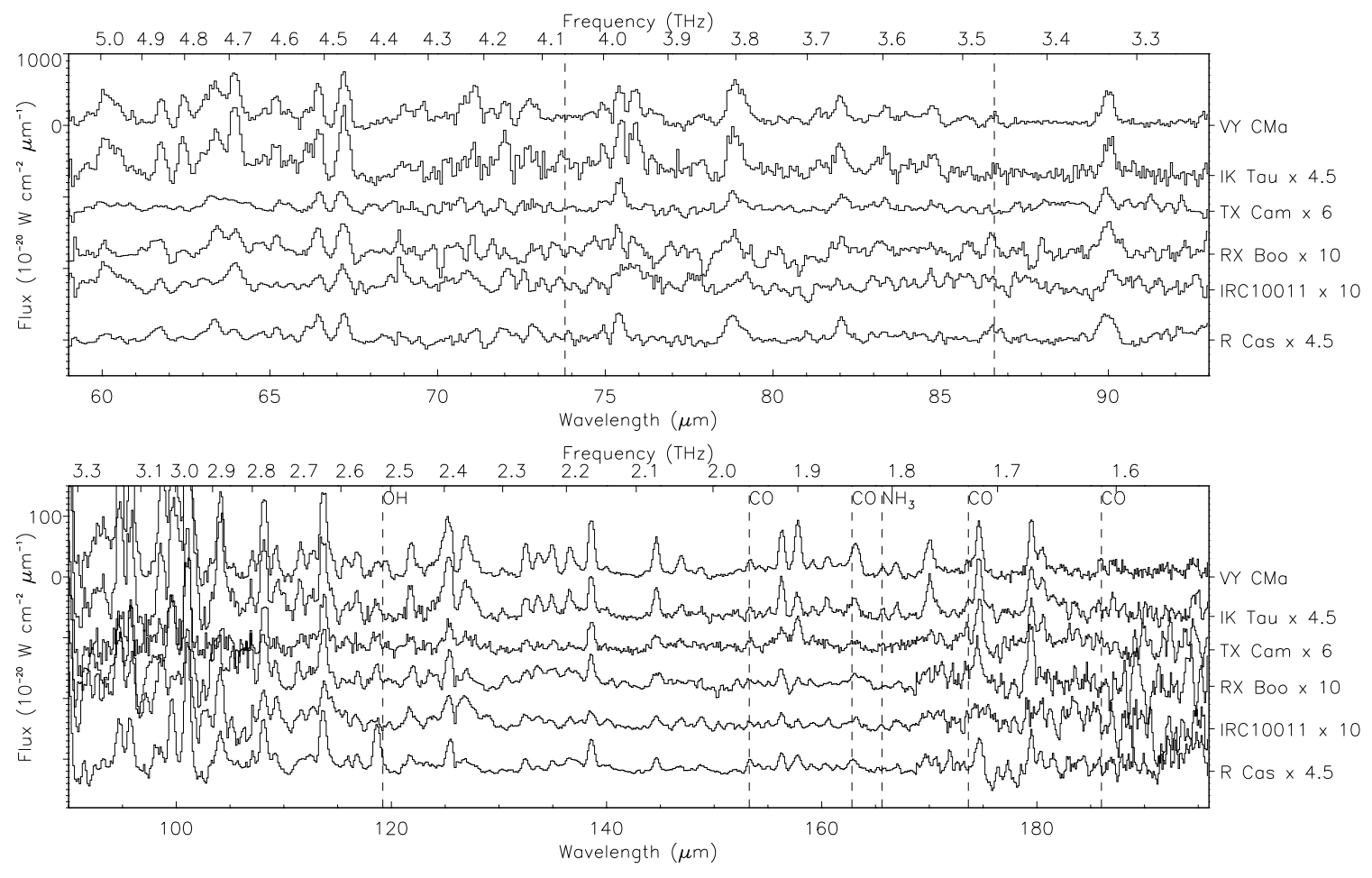

Fig. 6. Comparison of the spectrum of VY CMa with the other stars (the spectra of the other stars have been smoothed and shifted for clarity - see text).

Table 7. Results of fitting a straight line to the rotation diagram for all of the stars in our sample.

\begin{tabular}{cccc}
\hline \hline Star & $\begin{array}{c}T_{\text {rot }} \\
(\mathrm{K})\end{array}$ & $\begin{array}{c}N_{\text {tot }}\left(\mathrm{H}_{2} \mathrm{O}\right) \\
\left(10^{18} \mathrm{~cm}^{-2}\right)\end{array}$ & $\begin{array}{c}\text { Number of fitted } \\
\text { Data points }\end{array}$ \\
\hline VY CMa & $670_{-130}^{+210}$ & $70 \pm 12$ & 141 \\
IK Tau & $590_{-100}^{+150}$ & $1.5 \pm 0.3$ & 140 \\
TX Cam & $540_{-90}^{+140}$ & $3.7 \pm 0.9$ & 125 \\
RX Boo & $500_{-80}^{+110}$ & $3.0 \pm 0.6$ & 125 \\
IRC+10011 & $1400_{-500}^{+1500}$ & $5.6 \pm 1.3$ & 130 \\
R Cas & $650_{-150}^{+270}$ & $8.4 \pm 2.3$ & 132 \\
\hline
\end{tabular}

\section{Summary}

In this paper we present the ISO LWS spectrum of the luminous supergiant star VY CMa and give a brief description of the detected lines. We then compared the spectrum with several other evolved star spectra, particularly the Mira variable IK Tau.

The detected lines in the spectra of VY CMa and IK Tau are remarkably similar, both in the species detected and the relative intensity of the water lines.

We draw the following conclusions:

- We confirm that the spectra of our sample of evolved stars are dominated by water line emission in the FIR, and we report the detection of nearly all the $\mathrm{H}_{2} \mathrm{O}$ lines up to $\sim 2000 \mathrm{~K}$ above the ground state.

- A simple rotation analysis of the water lines shows that there are a probably a mixture of optically thick and thin lines which may be subthermally excited (the fit gives a rotational temperature of $670_{-130}^{+210} \mathrm{~K}$, and a corresponding column density $N_{\text {tot }}=(7.0 \pm 1.2) \times 10^{19} \mathrm{~cm}^{-2}$ which should be treated a lower limit to the true value).

- We tentatively assign several of the detected features to excited water in its $v_{2}=1$ vibrational state.
- We estimate the column density of $\mathrm{CO}$ to be $\left(9_{-7}^{+24}\right) \times$ $10^{17} \mathrm{~cm}^{-2}$, showing that water is the dominant oxygen carrier in these envelopes.

- We present a detection of ammonia in VY CMa and IK Tau, and a tentative detection of the $\mathrm{H}_{3} \mathrm{O}^{+}$ion toward VY CMa.

We note that the kinematics around VY CMa are very complex as shown by interferometric CO observations (Muller et al. 2007) which suggest the presence of spherically symmetric outflow together with a high velocity bipolar flow. The water maser observations by Menten et al. (2008) further imply that some fraction of the water emission may be emitted by special regions associated with shock fronts, excited in photochemical reactions associated with the outflow material. The presence of this complex excitation environment has also been indicated by observations of other molecules (Ziurys et al. 2009; Smith et al. 2009). To bring together an accurate understanding of the local environment will require a sophisticated model beyond the scope of the present study. Future observations with Herschel and ALMA should bring the necessary data to understand this complicated source. In particular, HIFI will add the ground state water lines, the isotopic $\mathrm{H}_{2}^{18} \mathrm{O}$ lines and the line profiles which may be used to study kinematics and self-absorption effects, and PACS will reobserve all of the lines above $55 \mu \mathrm{m}$. ALMA will give the spatial distribution of $\mathrm{H}_{2} \mathrm{O}$ and other molecules in these AGB envelopes.

Acknowledgements. E. T. P. would like to thank E. Krügel for help and encouragement in Bonn, and also M. Barlow for helpful advice and T. W. Grundy for useful discussions about the ISO L01 spectra and HPDP data. We made use of the Cologne Database for Molecular Spectroscopy (CDMS; Müller et al. 2005). The LWS Interactive Analysis (LIA) package is a joint development of the ISOLWS Instrument Team at the Rutherford Appleton Laboratory (RAL, UK - the PI Institute) and the Infrared Processing and Analysis Center (IPAC/Caltech, USA). The ISO Spectral Analysis Package (ISAP) is a joint development by the LWS and SWS Instrument Teams and Data Centres. Contributing institutes are CESR, IAS, IPAC, MPE, RAL and SRON. 
E. T. Polehampton et al.: The ISO LWS line spectrum of VY CMa

\section{Appendix A: Full list of fitted fluxes}

Table A.1. All line fluxes calculated by our fitting procedure for the 6 stars.

\begin{tabular}{|c|c|c|c|c|c|c|c|c|}
\hline Transition & $\begin{array}{l}\text { Wavelength } \\
(\mu \mathrm{m})\end{array}$ & $\begin{array}{c}\text { Energy of } \\
\text { upper } \\
\text { State }(K)\end{array}$ & $\begin{array}{c}\text { VY CMa } \\
\left(10^{-16} \mathrm{~W} \mathrm{~m}^{-2}\right)\end{array}$ & IK Tau & TX Cam & RX Boo & IRC +10011 & R Cas \\
\hline SW1 & & & & & & & & \\
\hline $6_{60}-7_{35}$ & 43.790 & 1175.0 & 146 & 29.7 & 7.2 & 5.0 & 18.1 & 11.1 \\
\hline $5_{41}-4_{32}$ & 43.893 & 550.4 & 49.2 & 11.5 & 1.3 & 5.6 & 3.6 & 5.4 \\
\hline $7_{43}-7_{16}$ & 44.048 & 1013.2 & 208 & 49.9 & 7.9 & 21.1 & 21.7 & 17.0 \\
\hline $5_{42}-4_{31}$ & 44.195 & 552.3 & 183 & 48.7 & 17.7 & 6.2 & 12.1 & 25.2 \\
\hline $8_{36}-7_{25}$ & 44.702 & 1125.7 & 174 & 46.1 & 7.3 & 9.7 & 19.1 & 7.6 \\
\hline $5_{23}-4_{14}$ & 45.112 & 323.5 & 323 & 102 & 18.8 & 33.4 & 19.0 & 32.5 \\
\hline $3_{31}-2_{02}$ & 46.484 & 100.8 & 200 & 52.3 & 11.4 & 13.8 & 19.7 & 27.4 \\
\hline $6_{42}-6_{15}$ & 46.539 & 781.1 & 55.3 & 22.4 & 2.2 & 5.7 & 5.0 & 5.2 \\
\hline $6_{33}-6_{06}$ & 46.544 & 642.7 & 13.0 & 4.4 & 0.49 & 1.1 & 0.82 & 1.1 \\
\hline $10_{29}-9_{18}$ & 46.608 & 1552.6 & 13.2 & 5.5 & 3.4 & 0.83 & 0 & 5.6 \\
\hline $7_{35}-6_{24}$ & 46.746 & 867.3 & 102 & 17.4 & 10.1 & 0.11 & 2.4 & 17.7 \\
\hline $4_{40}-4_{13}$ & 47.028 & 396.4 & 63.1 & 14.1 & 4.5 & 6.3 & 2.0 & 4.8 \\
\hline $10_{19}-9_{28}$ & 47.029 & 1554.4 & 4.0 & 1.0 & 0.26 & 0.47 & 0.24 & 0.30 \\
\hline $11_{111}-10_{010}$ & 47.040 & 1603.6 & 0 & 0 & 0 & 0 & 0 & 0 \\
\hline $11_{011}-10_{110}$ & 47.045 & 1603.6 & 117 & 30.0 & 7.5 & 14.9 & 8.2 & 9.4 \\
\hline $8_{44}-9_{19}$ & 47.267 & 1324.0 & 0 & 0 & 1.3 & 0 & 0 & 0 \\
\hline $5_{41}-5_{14}$ & 47.420 & 574.7 & 312 & 62.4 & 24.5 & 27.0 & 21.2 & 43.8 \\
\hline $7_{61}-8_{36}$ & 47.601 & 1447.6 & 115 & 16.1 & 8.1 & 16.4 & 2.0 & 17.2 \\
\hline $5_{32}-4_{23}$ & 47.973 & 432.2 & 304 & 64.4 & 23.7 & 23.1 & 11.2 & 37.7 \\
\hline $4_{40}-3_{31}$ & 49.282 & 410.4 & 6.2 & 0.12 & 0.01 & 4.1 & 0.02 & 0.02 \\
\hline $6_{61}-7_{34}$ & 49.334 & 1212.0 & 77.2 & 25.8 & 2.2 & 6.8 & 5.9 & 7.2 \\
\hline $4_{41}-3_{30}$ & 49.337 & 410.7 & 50.3 & 15.2 & 1.6 & 3.9 & 2.3 & 4.8 \\
\hline $6_{34}-5_{23}$ & 49.391 & 642.4 & 167 & 50.7 & 13.3 & 9.4 & 4.5 & 15.7 \\
\hline $9_{28}-8_{17}$ & 50.634 & 1270.3 & 260 & 77.3 & 9.9 & 10.1 & 16.4 & 13.8 \\
\hline \multicolumn{9}{|l|}{ SW2 } \\
\hline $9_{27}-8_{36}$ & 51.071 & 1447.6 & 267 & 62.1 & 15.1 & 17.5 & 9.2 & 12.8 \\
\hline $10_{110}-9_{09}$ & 51.445 & 1323.9 & 217 & 51.2 & 0.62 & 10.2 & 10.9 & 4.8 \\
\hline $10_{010}-9_{19}$ & 51.461 & 1324.0 & 0.20 & 0.05 & 0 & 1.0 & 0.01 & 1.7 \\
\hline $9_{18}-8_{27}$ & 51.685 & 1274.2 & 230 & 41.0 & 18.5 & 8.4 & 6.3 & 3.1 \\
\hline $5_{50}-6_{25}$ & 52.864 & 795.5 & 44.1 & 14.1 & 13.7 & 7.9 & 0 & 17.2 \\
\hline $5_{33}-4_{22}$ & 53.138 & 454.3 & 175 & 37.7 & 23.3 & 12.1 & 3.4 & 11.1 \\
\hline $7_{43}-8_{18}$ & 53.455 & 1070.7 & 24.9 & 12.8 & 29.3 & 10.9 & 0.70 & 8.3 \\
\hline $5_{32}-5_{05}$ & 54.507 & 468.1 & 158 & 60.6 & 21.6 & 9.2 & 16.0 & 14.5 \\
\hline $8_{27}-7_{16}$ & 55.131 & 1013.2 & 149 & 34.2 & 0.62 & 26.6 & 12.6 & 9.8 \\
\hline $10_{29}-10_{110}$ & 55.840 & 1603.6 & 0 & 0.40 & 0 & 6.6 & 2.0 & 0 \\
\hline $6_{51}-7_{26}$ & 55.858 & 1021.0 & 104 & 42.0 & 0.54 & 12.9 & 3.8 & 0 \\
\hline $10_{19}-10_{010}$ & 56.027 & 1603.6 & 66.8 & 1.9 & 1.5 & 0.03 & 0 & 0 \\
\hline $4_{31}-3_{22}$ & 56.325 & 296.8 & 155 & 27.4 & 0 & 14.9 & 0 & 0 \\
\hline $9_{19}-8_{08}$ & 56.771 & 1070.5 & 126 & 12.9 & 0.78 & 13.6 & 0 & 0 \\
\hline $9_{09}-8_{18}$ & 56.816 & 1070.7 & 13.4 & 3.9 & 0.90 & 0.94 & 0 & 0.78 \\
\hline $8_{53}-9_{28}$ & 56.972 & 1554.4 & 109 & 20.4 & 0.15 & 11.1 & 4.6 & 12.4 \\
\hline $7_{52}-8_{27}$ & 57.394 & 1274.2 & 73.4 & 9.2 & 7.2 & 6.5 & 0 & 9.7 \\
\hline $4_{22}-3_{13}$ & 57.637 & 204.7 & 142 & 29.4 & 10.6 & 21.6 & 6.1 & 14.6 \\
\hline $8_{17}-7_{26}$ & 57.709 & 1021.0 & 221 & 45.8 & 10.4 & 16.4 & 13.9 & 19.0 \\
\hline $6_{42}-7_{17}$ & 58.377 & 843.8 & 0 & 0.20 & 0 & 5.3 & 0 & 0 \\
\hline $4_{32}-3_{21}$ & 58.699 & 305.2 & 151 & 33.4 & 7.6 & 1.5 & 11.7 & 15.0 \\
\hline \multicolumn{9}{|l|}{ SW3 } \\
\hline $7_{26}-6_{15}$ & 59.987 & 781.1 & 76.4 & 17.7 & 3.8 & 4.8 & 8.0 & 1.8 \\
\hline $8_{26}-7_{35}$ & 60.162 & 1175.0 & 106 & 28.4 & 4.8 & 2.5 & 7.5 & 7.2 \\
\hline $7_{62}-8_{35}$ & 60.229 & 1511.0 & 8.4 & 0.90 & 0.08 & 0 & 1.8 & 0.18 \\
\hline $5_{41}-6_{16}$ & 61.316 & 643.5 & 10.5 & 0 & 0 & 0 & 0 & 1.0 \\
\hline $4_{31}-4_{04}$ & 61.809 & 319.5 & 95.2 & 24.8 & 4.9 & 4.5 & 2.5 & 11.7 \\
\hline $4_{40}-5_{15}$ & 61.916 & 469.9 & 1.9 & 0.49 & 0 & 0.06 & 0.05 & 0.06 \\
\hline $9_{36}-8_{45}$ & 62.418 & 1615.3 & 10.7 & 3.6 & 0.46 & 0.31 & 0.28 & 0.49 \\
\hline $9_{28}-9_{19}$ & 62.432 & 1324.0 & 103 & 27.8 & 2.0 & 0.41 & 2.9 & 1.4 \\
\hline $9_{18}-9_{09}$ & 62.928 & 1324.0 & 68.6 & 16.4 & 1.8 & 0 & 5.5 & 6.2 \\
\hline $8_{18}-7_{07}$ & 63.324 & 843.5 & 71.5 & 13.1 & 3.9 & 2.9 & 0 & 11.7 \\
\hline $8_{08}-7_{17}$ & 63.458 & 843.8 & 139 & 35.7 & 7.2 & 11.6 & 3.6 & 13.7 \\
\hline $7_{62}-7_{53}$ & 63.880 & 1524.6 & 12.1 & 2.7 & 0.01 & 0.01 & 0.23 & 0.19 \\
\hline
\end{tabular}


Table A.1. continued.

\begin{tabular}{|c|c|c|c|c|c|c|c|c|}
\hline Transition & $\begin{array}{c}\text { Wavelength } \\
\quad(\mu \mathrm{m})\end{array}$ & $\begin{array}{c}\text { Energy of } \\
\text { upper } \\
\text { State }(\mathrm{K})\end{array}$ & $\begin{array}{c}\text { VY CMa } \\
\left(10^{-16} \mathrm{~W} \mathrm{~m}^{-2}\right)\end{array}$ & IK Tau & TX Cam & RX Boo & IRC+10011 & $\mathrm{R}$ Cas \\
\hline $6_{61}-6_{52}$ & 63.914 & 1278.5 & 88.9 & 25.3 & 3.1 & 6.2 & 4.5 & 5.0 \\
\hline $6_{60}-6_{51}$ & 63.928 & 1278.6 & 5.5 & 1.6 & 0.20 & 0.34 & 0.30 & 0.30 \\
\hline $7_{61}-7_{52}$ & 63.955 & 1524.9 & 110 & 32.4 & 4.3 & 5.2 & 6.0 & 5.0 \\
\hline $6_{25}-5_{14}$ & 65.166 & 574.7 & 107 & 24.2 & 1.3 & 8.3 & 3.6 & 10.1 \\
\hline $7_{16}-6_{25}$ & 66.093 & 795.5 & 60.3 & 27.2 & 2.7 & 2.2 & 0.84 & 8.6 \\
\hline $3_{30}-2_{21}$ & 66.438 & 194.1 & 156 & 37.8 & 10.3 & 11.8 & 5.2 & 20.7 \\
\hline $3_{31}-2_{20}$ & 67.089 & 195.9 & 87.7 & 20.9 & 11.0 & 7.0 & 7.7 & 8.1 \\
\hline $3_{30}-3_{03}$ & 67.269 & 196.8 & 153 & 41.7 & 6.3 & 10.3 & 5.5 & 18.5 \\
\hline \multicolumn{9}{|l|}{ SW4 } \\
\hline $8_{27}-8_{18}$ & 70.703 & 1070.7 & 81.7 & 7.5 & 6.6 & 3.6 & 7.7 & 2.2 \\
\hline $5_{24}-4_{13}$ & 71.067 & 396.4 & 145 & 25.9 & 3.2 & 8.3 & 3.0 & 3.6 \\
\hline $7_{17}-6_{06}$ & 71.540 & 642.7 & 56.2 & 5.8 & 1.2 & 3.4 & 0 & 0 \\
\hline $5_{51}-6_{24}$ & 71.788 & 867.3 & 1.10 & 0.57 & 0 & 0 & 0.09 & 2.1 \\
\hline $7_{07}-6_{16}$ & 71.947 & 643.5 & 85.4 & 30.4 & 5.4 & 2.2 & 4.4 & 10.6 \\
\hline $8_{17}-8_{08}$ & 72.032 & 1070.5 & 18.3 & 10.3 & 0 & 0.81 & 6.2 & 0 \\
\hline $9_{37}-9_{28}$ & 73.613 & 1554.4 & 37.1 & 15.8 & 0 & 2.0 & 6.6 & 1.4 \\
\hline $7_{25}-6_{34}$ & 74.945 & 933.7 & 81.0 & 25.6 & 3.7 & 5.3 & 6.1 & 12.7 \\
\hline $3_{21}-2_{12}$ & 75.381 & 114.4 & 94.8 & 23.0 & 10.9 & 10.2 & 2.3 & 14.7 \\
\hline $8_{54}-8_{45}$ & 75.496 & 1615.3 & 68.2 & 30.6 & 6.6 & 3.8 & 6.5 & 12.7 \\
\hline $5_{51}-5_{42}$ & 75.781 & 877.8 & 47.2 & 13.0 & 0.69 & 6.7 & 5.0 & 0 \\
\hline $7_{53}-7_{44}$ & 75.813 & 1334.8 & 18.7 & 4.7 & 0.16 & 2.9 & 1.7 & 0 \\
\hline $6_{52}-6_{43}$ & 75.830 & 1088.8 & 0.03 & 0.01 & 0 & 0 & 0 & 0 \\
\hline $5_{50}-5_{41}$ & 75.910 & 878.2 & 93.5 & 33.8 & 2.5 & 0.84 & 5.3 & 5.2 \\
\hline $6_{51}-6_{42}$ & 76.422 & 1090.3 & 50.9 & 15.9 & 0 & 0 & 3.9 & 5.3 \\
\hline $7_{52}-7_{43}$ & 77.761 & 1339.9 & 37.4 & 10.1 & 0 & 4.2 & 3.9 & 0 \\
\hline \multicolumn{9}{|l|}{ SW5 } \\
\hline $4_{23}-3_{12}$ & 78.742 & 249.4 & 110 & 23.2 & 11.2 & 4.0 & 1.7 & 12.6 \\
\hline $6_{15}-5_{24}$ & 78.928 & 598.8 & 125 & 26.1 & 4.8 & 7.8 & 4.7 & 9.8 \\
\hline $9_{46}-9_{37}$ & 80.222 & 1749.9 & 32.3 & 6.3 & 2.9 & 5.2 & 0 & 0 \\
\hline $8_{53}-8_{44}$ & 80.557 & 1628.4 & 28.7 & 6.2 & 1.1 & 0 & 0 & 0 \\
\hline $7_{26}-7_{17}$ & 81.216 & 843.8 & 34.0 & 4.8 & 2.0 & 0 & 0.84 & 0.32 \\
\hline $9_{27}-9_{18}$ & 81.405 & 1552.6 & 43.2 & 10.2 & 1.7 & 0 & 0.06 & 0 \\
\hline $88_{35}-7_{44}$ & 81.690 & 1334.8 & 27.6 & 2.4 & 0.09 & 4.1 & 2.0 & 0 \\
\hline $6_{16}-5_{05}$ & 82.031 & 468.1 & 110 & 24.8 & 8.8 & 9.4 & 1.4 & 16.0 \\
\hline $8_{36}-8_{27}$ & 82.977 & 1274.2 & 35.3 & 9.0 & 5.0 & 4.6 & 5.6 & 6.8 \\
\hline $6_{06}-5_{15}$ & 83.284 & 469.9 & 60.0 & 17.0 & 4.9 & 3.7 & 0.50 & 6.0 \\
\hline $7_{16}-7_{07}$ & 84.767 & 843.5 & 75.2 & 16.9 & 5.1 & 6.7 & 7.6 & 3.8 \\
\hline $8_{45}-8_{36}$ & 85.769 & 1447.6 & 36.5 & 3.4 & 0 & 4.1 & 5.1 & 0 \\
\hline $3_{22}-2_{11}$ & 89.988 & 136.9 & 85.4 & 19.0 & 8.1 & 7.5 & 2.9 & 10.0 \\
\hline $7_{44}-7_{35}$ & 90.050 & 1175.0 & 50.1 & 10.8 & 2.0 & 10.4 & 5.3 & 11.8 \\
\hline \multicolumn{9}{|l|}{ LW1 } \\
\hline $6_{43}-6_{34}$ & 92.811 & 933.7 & 43.2 & 5.2 & 0 & 3.0 & 3.5 & 0 \\
\hline $7_{35}-7_{26}$ & 93.383 & 1021.0 & 40.3 & 4.6 & 1.1 & 0 & 4.2 & 3.0 \\
\hline $6_{52}-7_{25}$ & 94.172 & 1125.7 & 32.8 & 5.0 & 1.7 & 0 & 3.9 & 1.8 \\
\hline $5_{42}-5_{33}$ & 94.210 & 725.1 & 3.3 & 0.66 & 1.2 & 0 & 0.35 & 0.20 \\
\hline $6_{25}-6_{16}$ & 94.644 & 643.5 & 43.4 & 8.1 & 1.3 & 1.9 & 2.3 & 2.9 \\
\hline $4_{41}-4_{32}$ & 94.705 & 550.4 & 65.3 & 13.7 & 3.0 & 3.7 & 4.0 & 7.3 \\
\hline $9_{45}-8_{54}$ & 95.176 & 1805.9 & 46.6 & 9.3 & 1.9 & 3.7 & 2.9 & 1.8 \\
\hline $5_{15}-4_{04}$ & 95.627 & 319.5 & 73.7 & 14.0 & 3.4 & 4.9 & 4.5 & 10.8 \\
\hline $4_{40}-4_{31}$ & 95.885 & 552.3 & 59.1 & 13.6 & 3.9 & 2.3 & 3.1 & 2.8 \\
\hline $9_{37}-10_{010}$ & 98.329 & 1603.6 & 45.8 & 9.3 & 2.1 & 2.3 & 3.7 & 2.3 \\
\hline $5_{41}-5_{32}$ & 98.494 & 732.1 & 54.8 & 7.7 & 1.1 & 0.56 & 3.7 & 3.2 \\
\hline $5_{05}-4_{14}$ & 99.493 & 323.5 & 85.2 & 18.8 & 6.4 & 6.0 & 3.9 & 15.4 \\
\hline $8_{26}-8_{17}$ & 99.979 & 1270.3 & 92.4 & 22.0 & 2.8 & 3.6 & 3.9 & 6.1 \\
\hline $5_{14}-4_{23}$ & 100.913 & 432.2 & 89.3 & 23.6 & 6.0 & 5.5 & 3.7 & 11.2 \\
\hline $2_{20}-1_{11}$ & 100.983 & 53.4 & 105 & 25.4 & 5.9 & 7.2 & 3.9 & 14.3 \\
\hline $6_{24}-5_{33}$ & 101.209 & 725.1 & 84.4 & 21.2 & 5.4 & 3.5 & 2.8 & 8.7 \\
\hline $6_{42}-6_{33}$ & 103.916 & 951.8 & 52.8 & 15.7 & 2.4 & 4.3 & 3.6 & 5.9 \\
\hline $6_{15}-6_{06}$ & 103.940 & 642.7 & 0.56 & 0.17 & 0.03 & 0.85 & 0.03 & 0.07 \\
\hline $6_{34}-6_{25}$ & 104.094 & 795.5 & 35.5 & 8.1 & 3.4 & 0.85 & 1.7 & 3.7 \\
\hline
\end{tabular}


Table A.1. continued.

\begin{tabular}{|c|c|c|c|c|c|c|c|c|}
\hline Transition & $\begin{array}{l}\text { Wavelength } \\
\qquad(\mu \mathrm{m})\end{array}$ & $\begin{array}{l}\text { Energy of } \\
\text { upper } \\
\text { State }(\mathrm{K})\end{array}$ & $\begin{array}{c}\text { VY CMa } \\
\left(10^{-16} \mathrm{~W} \mathrm{~m}^{-2}\right)\end{array}$ & IK Tau & TX Cam & RX Boo & IRC+10011 & R Cas \\
\hline \multicolumn{9}{|l|}{ LW2 } \\
\hline $22_{21}-1_{10}$ & 108.073 & 61.0 & 70.6 & 18.2 & 9.7 & 6.3 & 4.1 & 15.3 \\
\hline $5_{24}-5_{15}$ & 111.628 & 469.9 & 30.0 & 8.5 & 3.6 & 0.74 & 2.2 & 3.4 \\
\hline $7_{43}-7_{34}$ & 112.511 & 1212.0 & 21.8 & 5.1 & 1.5 & 1.9 & 2.1 & 4.0 \\
\hline $4_{41}-5_{14}$ & 112.803 & 574.7 & 12.7 & 3.0 & 0.89 & 1.7 & 1.6 & 1.7 \\
\hline $4_{14}-3_{03}$ & 113.537 & 196.8 & 59.2 & 15.7 & 5.1 & 3.6 & 3.0 & 13.3 \\
\hline $5_{33}-5_{24}$ & 113.948 & 598.8 & 59.1 & 13.8 & 5.5 & 5.1 & 4.1 & 10.7 \\
\hline $9_{27}-10_{110}$ & 114.454 & 1603.6 & 11.7 & 5.1 & 0.76 & 1.1 & 2.8 & 1.9 \\
\hline $8_{36}-9_{09}$ & 116.350 & 1323.9 & 10.2 & 2.2 & 0 & 0.04 & 0.92 & 0.19 \\
\hline $7_{34}-6_{43}$ & 116.779 & 1088.8 & 21.3 & 6.0 & 0.35 & 2.0 & 1.4 & 4.8 \\
\hline $9_{46}-8_{53}$ & 117.684 & 1807.0 & 1.7 & 0 & 0 & 0.19 & 0.52 & 0.36 \\
\hline $9_{37}-8_{44}$ & 118.405 & 1628.4 & 11.0 & 3.1 & 1.2 & 1.3 & 0.81 & 10.7 \\
\hline $4_{32}-4_{23}$ & 121.722 & 432.2 & 30.0 & 7.3 & 1.7 & 1.7 & 2.8 & 4.5 \\
\hline $8_{44}-8_{35}$ & 122.522 & 1511.0 & 10.2 & 2.2 & 0.27 & 0 & 0.55 & 1.8 \\
\hline $9_{36}-9_{27}$ & 123.460 & 1729.3 & 10.7 & 2.0 & 0.22 & 1.5 & 1.7 & 1.8 \\
\hline $4_{04}-3_{13}$ & 125.354 & 204.7 & 51.8 & 15.3 & 4.3 & 4.0 & 4.0 & 7.3 \\
\hline \multicolumn{9}{|l|}{ LW3 } \\
\hline $3_{31}-3_{22}$ & 126.714 & 296.8 & 33.0 & 8.9 & 3.5 & 0.76 & 4.1 & 4.4 \\
\hline $7_{25}-7_{16}$ & 127.884 & 1013.2 & 14.8 & 4.0 & 2.7 & 0.78 & 2.4 & 1.5 \\
\hline $9_{45}-9_{36}$ & 129.339 & 1845.8 & 3.0 & 0.38 & 0.61 & 0 & 1.4 & 0 \\
\hline $7_{53}-8_{26}$ & 130.319 & 1414.2 & 8.4 & 2.3 & 1.0 & 0.85 & 0.45 & 0.41 \\
\hline $4_{23}-4_{14}$ & 132.408 & 323.5 & 30.1 & 4.9 & 2.5 & 2.4 & 1.8 & 4.3 \\
\hline $8_{36}-7_{43}$ & 133.549 & 1339.9 & 21.0 & 5.4 & 2.0 & 3.3 & 1.6 & 1.7 \\
\hline $5_{14}-5_{05}$ & 134.935 & 468.1 & 30.3 & 5.8 & 2.0 & 2.7 & 0.99 & 3.5 \\
\hline $3_{30}-3_{21}$ & 136.496 & 305.2 & 27.6 & 7.3 & 2.8 & 3.1 & 1.6 & 4.0 \\
\hline $7_{35}-8_{08}$ & 137.683 & 1070.5 & 6.6 & 2.1 & 0.92 & 1.4 & 1.2 & 1.8 \\
\hline $3_{13}-2_{02}$ & 138.528 & 100.8 & 45.1 & 9.3 & 6.0 & 5.4 & 1.8 & 8.1 \\
\hline $8_{44}-7_{53}$ & 138.641 & 1524.6 & 11.4 & 2.4 & 1.1 & 1.0 & 0.53 & 2.1 \\
\hline $4_{13}-3_{22}$ & 144.518 & 296.8 & 32.2 & 7.4 & 3.1 & 2.4 & 1.4 & 3.7 \\
\hline $4_{31}-4_{22}$ & 146.923 & 454.3 & 19.7 & 2.9 & 2.3 & 0.9 & 1.3 & 1.5 \\
\hline \multicolumn{9}{|l|}{ LW4 } \\
\hline $8_{35}-8_{26}$ & 148.708 & 1414.2 & 5.6 & 0.83 & 0.65 & 1.4 & 0.73 & 0.71 \\
\hline $5_{42}-6_{15}$ & 148.790 & 781.1 & 3.6 & 1.4 & 0.29 & 0.22 & 0.65 & 0.69 \\
\hline $3_{22}-3_{13}$ & 156.194 & 204.7 & 5.7 & 1.5 & 0.75 & 0.67 & 0.21 & 0.54 \\
\hline $5_{23}-4_{32}$ & 156.265 & 550.4 & 37.0 & 10.0 & 2.9 & 1.7 & 1.6 & 3.4 \\
\hline $3_{31}-4_{04}$ & 158.312 & 319.5 & 13.4 & 0.48 & 1.6 & 0.33 & 0.19 & 0.83 \\
\hline $8_{45}-7_{52}$ & 159.051 & 1524.9 & 10.4 & 1.8 & 0.81 & 0.59 & 0.73 & 1.2 \\
\hline $6_{34}-7_{07}$ & 159.400 & 843.5 & 6.8 & 1.2 & 0.79 & 0.20 & 0.45 & 0.49 \\
\hline $8_{26}-9_{19}$ & 159.485 & 1324.0 & 0.76 & 0.25 & 0.70 & 0.01 & 0.14 & 0.09 \\
\hline $5_{32}-5_{23}$ & 160.510 & 642.4 & 18.7 & 5.5 & 2.6 & 0.09 & 1.2 & 1.6 \\
\hline $7_{34}-7_{25}$ & 166.815 & 1125.7 & 5.2 & 0.58 & 0 & 0.06 & 0.33 & 0.17 \\
\hline $6_{24}-6_{15}$ & 167.035 & 781.1 & 10.9 & 3.7 & 0.80 & 0.26 & 1.0 & 0.82 \\
\hline \multicolumn{9}{|l|}{ LW5 } \\
\hline $7_{35}-6_{42}$ & 169.739 & 1090.3 & 15.5 & 0.19 & 0.46 & 0 & 1.4 & 4.1 \\
\hline $6_{33}-6_{24}$ & 170.139 & 867.3 & 35.4 & 9.9 & 2.2 & 0.55 & 2.1 & 1.4 \\
\hline $5_{33}-6_{06}$ & 174.607 & 642.7 & 48.8 & 14.4 & 4.3 & 4.9 & 2.6 & 6.7 \\
\hline $3_{03}-2_{12}$ & 174.626 & 114.4 & 3.2 & 1.4 & 0.34 & 0.25 & 0.10 & 0.71 \\
\hline $4_{32}-5_{05}$ & 174.920 & 468.1 & 5.7 & 0.10 & 1.6 & 0.63 & 2.3 & 0.60 \\
\hline $22_{12}-1_{01}$ & 179.527 & 34.2 & 52.7 & 11.2 & 5.0 & 5.5 & 0 & 8.8 \\
\hline $2_{21}-2_{12}$ & 180.488 & 114.4 & 26.4 & 8.5 & 2.1 & 0.26 & 3.7 & 4.2 \\
\hline $4_{13}-4_{04}$ & 187.111 & 319.5 & 15.1 & 4.9 & 0 & 0 & 0.84 & 1.3 \\
\hline $8_{54}-9_{27}$ & 187.810 & 1729.3 & 3.7 & 0.91 & 0 & 1.8 & 0.43 & 3.6 \\
\hline $6_{43}-7_{16}$ & 190.437 & 1013.2 & 6.8 & 0.64 & 1.4 & 0 & 2.3 & 1.3 \\
\hline $6_{33}-5_{42}$ & 194.422 & 877.8 & 14.6 & 4.4 & 1.5 & 4.8 & 0.88 & 7.9 \\
\hline
\end{tabular}

\section{References}

Barlow, M. J. 1999, in Asymptotic Giant Branch Stars, ed. T. Le Bertre, A. Lèbre, \& C. Waelkens, IAU Symp., 191, 353

Barlow, M. J., Nguyen-Q-Rieu, Truong-Bach, et al. 1996, A\&A, 315, L241 Choi, Y. K., Hirota, T., Honma, M., et al. 2008, PASJ, 60, 1007

Clegg, P. E., Ade, P. A. R., Armand, C., et al. 1996, A\&A, 315, L38 de Graaw, T., Haser, L. N., Beintema, D. A., et al. 1996, A\&A, 315, L49

Decin, L., Hony, S., de Koter, A., et al. 2006, A\&A, 456, 549
Decin, L., Hony, S., de Koter, A., et al. 2007, A\&A, 475, 233 Goicoechea, J. R., \& Cernicharo, J. 2001, ApJ, 554, L213

Goldsmith, P. F., \& Langer, W. D. 1999, ApJ, 517, 209

Gry, C., Swinyard, B., Harwood, A., et al. 2003, ISO Handbook Volume III (LWS), Version 2.1, ESA SAI-99-077/Dc

Harwit, M., Malfait, K., Decin, L., et al. 2001, ApJ, 557, 844

Justtanont, K., Skinner, C. J., \& Tielens, A. G. G. M. 1994, ApJ, 435, 852

Kemper, F., Stark, R., Justtanont, K., et al. 2003, A\&A, 407, 609

Kessler, M. F., Steinz, J. A., Anderegg, M. E., et al. 1996, A\&A, 315, L27 
Lada, C. J., \& Reid, M. J. 1978, ApJ, 219, 95

Lim, T. L., Hutchinson, G., Sidher, S. D., et al. 2002, SPIE, 4847, 435

Liu, D.-J., Oka, T., \& Sears, T. J. 1986, J. Chem. Phys., 84, 1312

Lloyd, C., Lerate, M. R., \& Grundy, T. W. 2003, The LWS L01 Pipeline, version 1, available from the ISO Data Archive at

http: //www.iso.vilspa.esa.es/ida/

Maercker, M., Schöier, F. L., Olofsson, H., Bergman, P., \& Ramstedt, S. 2008, A\&A, 479, 779

Mamon, G. A., Glassgold, A. E., \& Omont, A. 1987, ApJ, 323, 306

McLaren, R. A., \& Betz, A. L. 1980, ApJ, 240, L159

Menten, K. M., \& Alcolea, J. 1995, ApJ, 448, 416

Menten, K. M., \& Melnick, G. J. 1989, ApJ, 341, 91

Menten, K. M., Philipp, S. D., Güsten, R., et al. 2006, A\&A, 454, L107

Menten, K. M., Lundgren, A., Belloche, A., Thorwirth, S., \& Reid, M. 2008, A\&A, 477, 185

Monnier, J. D., Danchi, W. C., Hale, D. S., Tuthill, P. G., \& Townes, C. H. 2000 ApJ, 543, 868

Müller, H. S. P., Schlöder, F., Stutzki, J., et al. 2005, J. Mol. Struct., 742, 215

Muller, S., Dinh-V-Trung; Lim, J., Hirano, N., \& Muthu, C., Kwok, S. 2007, ApJ, 656, 1109
Neufeld, D. A., Chen, W., Melnick, G. J., et al. 1996, A\& A, 315, L237

Neufeld, D. A., Feuchtgruber, H., Harwit, M., et al. 1999, ApJ, 517, L147

Olivier, E. A., Whitelock, P., \& Marang, F. 2001, MNRAS, 326, 490

Phillips, T. G., van Dishoeck, E. F., \& Keene, J. 1992, ApJ, 399, 533

Pickett, H. M., Poynter, R. L., Cohen, E. A., et al. 1998, J. Quant. Spectrosc. \& Rad. Transfer, 60, 883

Polehampton, E. T., Baluteau, J.-P., Swinyard, B. M., et al. 2007, MNRAS, 377, 1122

Smith, N., Hinkle, K., \& Ryde, N. 2009, AJ, 137, 3558

Sturm, E., Bauer, O. H., Lutz, D., et al. 1998, in Astronomical Data Analysis Software and Systems VII, ASP Conf. Ser., 145, 161

Sylvester, R. J., Barlow, M. J., Nguyen-Q-Rieu, et al. 1997, MNRAS, 291, L42

Teyssier, D., Hernandez, R., Bujarrabal, V., Yoshida, H., \& Phillips, T. G. 2006, A\&A, 450, 167

Truong-Bach, Sylvester, R. J., Barlow, M. J., et al. 1999, A\&A, 345, 925

van der Tak, F. F. S., Belloche, A., Schilke, P., et al. 2006, A\&A, 454, L99

Willacy, K., \& Millar, T. J. 1997, A\&A, 324, 237

Wootten, A., Mangum, J. G., Turner, B. E., et al. 1991, ApJ, 380, L79

Ziurys, L., Tenenbaum, E., Pulliam, R., Woolf, N., \& Milam, S. 2009, ApJ, 695, 1604 